\title{
Adaptive stabilized finite volume method and convergence analysis for the Oseen equations
}

\author{
Junxiang $\mathrm{Lu}^{1 *}$ and Tong Zhang ${ }^{2^{*}}$
}

\section{"Correspondence:}

45621969@qq.com;

zhangtong0616@163.com

'School of Science, Xi'an

Polytechnic University, Xi'an, China

${ }^{2} S$ chool of Mathematics \&

Information Science, Henan

Polytechnic University, Jiaozuo,

China

\begin{abstract}
In this paper, based on the pressure project method, we consider an adaptive stabilized finite volume method for the Oseen equations with the lowest equal order finite element pair. Firstly, we develop the discrete forms in both finite element and finite volume methods, and establish the existence and uniqueness of numerical solutions by establishing the equivalence of linear terms in finite element and finite volume methods. Secondly, a residual type a posteriori error estimator is designed, and the computable global upper and local lower bounds between the exact solutions and the finite volume solutions are established. Thirdly, a discrete local lower bound between two successive finite volume solutions is obtained, convergence analysis of the adaptive stabilized finite volume method is also performed. Finally, some numerical results are presented to verify the performances of the developed error estimators and confirm the established theoretical findings.
\end{abstract}

MSC: 65N15; 65N30; 76D05

Keywords: Adaptive algorithm; Stabilized finite volume method; Oseen equations; Convergence

\section{Introduction}

Finite volume method, as an important numerical tool for solving partial differential equations, has been widely used in the engineering community for fluid computations (see [9, $14,15,29,30])$. Finite volume method is intuitive since it is based on local conservation of mass, momentum, and energy over volumes. Finite volume method has a flexibility similar to that of the finite element method for handling complicated geometries, and its implementation is comparable to that of the finite difference method. Furthermore, its numerical solution usually has certain conservation features that are desirable in many practical applications. Based on the above reasons, several researchers have contributed to this method extensively and obtained numerous important results. For example, we can refer to $[14,26]$ for the monographs, and for the recent developments about the finite volume method, we can read $[1,8,13,20,31,37]$ and the references therein.

Let $\Omega \in \mathbb{R}^{2}$ be a bounded polygon domain with Lipschitz continuous boundary $\partial \Omega$. We consider the following Oseen problem, which consists of finding a pair $(\mathbf{u}, p)$ as the solution

(c) The Author(s) 2018. This article is distributed under the terms of the Creative Commons Attribution 4.0 International License (http://creativecommons.org/licenses/by/4.0/), which permits unrestricted use, distribution, and reproduction in any medium, provided you give appropriate credit to the original author(s) and the source, provide a link to the Creative Commons license, and indicate if changes were made. 
of the equations

$$
\begin{cases}-v \Delta \mathbf{u}+\mathbf{a} \cdot \nabla \mathbf{u}+\nabla p=\mathbf{f} & \text { in } \Omega, \\ \nabla \cdot \mathbf{u}=0 & \text { in } \Omega, \\ \mathbf{u}=\mathbf{0} & \text { on } \partial \Omega,\end{cases}
$$

where $\mathbf{u}$ is the velocity field, $p$ is the pressure, $v$ is the viscosity, $\mathbf{f}$ is the body forces. For the sake of simplicity, we consider the simplest Dirichlet condition. Several simplifying assumptions will be made for the advection vector $\mathbf{a}$. In particular, we take $\mathbf{a} \in C^{0}(\bar{\Omega})$, weakly divergence free with derivatives of order up to $m$ is locally bounded $(m>0$ is integer, see Assumption A1 in Sect. 2).

The Oseen problem stated above can be considered as a linearization of the stationary incompressible Navier-Stokes equations. It also appears as one of the steps of some multilevel methods for these equations, or results from a time discretization of the transient Navier-Stokes problem if the advection coefficient $\mathbf{a}$ is treated explicitly. This is why it is often used as a first step towards the analysis of the full nonlinear problem, to obtain both a priori and a posteriori estimates.

Let us introduce some standard notations. The space of square integrable functions in $\Omega$ is denoted by $L^{2}(\Omega)$, and the space of functions whose distributional derivatives of order up to $m \geq 0$ belong to $L^{2}(\Omega)$ is denoted by $H^{m}(\Omega)$. The space $H_{0}^{1}(\Omega)$ consists of functions in $H^{1}(\Omega)$ vanishing on $\partial \Omega$. A bold character is used to denote the vector counterpart of all these spaces. The $L^{2}$ inner product in $\Omega$ is denoted by $(\cdot, \cdot)_{\Omega}$, and the norm in a Banach space $X$ by $\|\cdot\|_{X}$. This notation is simplified in some cases as follows: $(\cdot, \cdot)_{\Omega} \equiv(\cdot, \cdot)$, $\|\cdot\|_{L^{2}(\Omega)} \equiv\|\cdot\|_{0}$, for the positive integer $m$, set $\|\cdot\|_{H^{m}(\Omega)} \equiv\|\cdot\|_{m}$, and if $K$ is an element $\|\cdot\|_{L^{2}(K)} \equiv\|\cdot\|_{K},\|\cdot\|_{H^{m}(K)} \equiv\|\cdot\|_{m, K}$.

With above notations, the velocity and pressure spaces for the continuous problem are $X:=\mathbf{H}_{0}^{1}(\Omega), M:=L^{2}(\Omega) / \mathbb{R}$. The variational formulation for problem (1.1) reads as follows: For all $(\mathbf{v}, q) \in X \times M$, find $(\mathbf{u}, p) \in X \times M$ such that

$$
\left\{\begin{array}{l}
v(\nabla \mathbf{u}, \nabla \mathbf{v})-(\nabla \cdot \mathbf{v}, p)+(\mathbf{a} \cdot \nabla \mathbf{u}, \mathbf{v})=(\mathbf{f}, \mathbf{v}) \\
(\nabla \cdot \mathbf{u}, q)=0 .
\end{array}\right.
$$

The bilinear terms satisfy the following continuity and inf-sup properties (see [16]):

$$
\begin{aligned}
& |v(\nabla \mathbf{u}, \nabla \mathbf{v})-(\nabla \cdot \mathbf{v}, p)+(\mathbf{a} \cdot \nabla \mathbf{u}, \mathbf{v})+(\nabla \cdot \mathbf{u}, q)| \\
& \quad \leq C\left(\|\nabla \mathbf{u}\|_{0}+\|p\|_{0}\right)\left(\|\nabla \mathbf{v}\|_{0}+\|q\|_{0}\right), \\
& \beta\left(\|\nabla \mathbf{u}\|_{0}+\|p\|_{0}\right) \leq \sup _{0 \neq(\mathbf{v}, q) \in X \times M} \frac{|v(\nabla \mathbf{u}, \nabla \mathbf{v})-(\nabla \cdot \mathbf{v}, p)+(\mathbf{a} \cdot \nabla \mathbf{u}, \mathbf{v})+(\nabla \cdot \mathbf{u}, q)|}{\|\nabla \mathbf{v}\|_{0}+\|q\|_{0}} .
\end{aligned}
$$

Here and below, $C>0$ is a generic constant depending at most on the data $\Omega, v$, a, and $\mathbf{f}$. In the following sections, $C_{1}, C_{2}, \ldots$ denote some positive constants depending only on $\Omega$.

From the above properties, we know that problem (1.2) is well posed.

The standard Galerkin approximation of problem (1.2) is straightforward. Let $\mathcal{T}_{H}$ denote a shape regular and conforming triangulation of the domain $\Omega$. The diameter of an element domain $K \in \mathcal{T}_{H}$ is denoted by $H_{K}$ and the diameter of the finite element partitions by $H=$ 
$\max \left\{H_{K} \mid K \in \mathcal{T}_{H}\right\}$. For simplicity, we assume that all the element domains are the image of a reference element $\widehat{K}$ through a polynomial mapping. We define the polynomial spaces $P_{k}(\widehat{K})$ with order $k$ on the element $\widehat{K}$. From these polynomial spaces, we construct the finite element spaces $X_{H} \subset X$ and $M_{H} \subset M$ in the usual manner. The discrete version of problem (1.2) is as follows: Find $\left(\mathbf{u}_{H}, p_{H}\right) \in X_{H} \times M_{H}$ such that

$$
\begin{cases}v\left(\nabla \mathbf{u}_{H}, \nabla \mathbf{v}_{H}\right)-\left(\nabla \cdot \mathbf{v}_{H}, p_{H}\right)+\left(\mathbf{a} \cdot \nabla \mathbf{u}_{H}, \mathbf{v}_{H}\right)=\left(\mathbf{f}, \mathbf{v}_{H}\right), & \forall \mathbf{v}_{H} \in X_{H}, \\ \left(\nabla \cdot \mathbf{u}_{H}, q_{H}\right)=0, & \forall q_{H} \in M_{H} .\end{cases}
$$

The well-posedness of problem (1.4) relies on the ellipticity of the viscous term and the inf-sup or the Babuška-Brezzi condition (see $[10,16]$ ), which have been shown to hold for the continuous problem. The first property is automatically inherited by its discrete counterpart. However, the inf-sup condition needs to be explicitly required. This leads to the need to use mixed interpolations and verify

$$
\beta_{*} \leq \inf _{0 \neq q_{H} \in M_{H}} \sup _{0 \neq \mathbf{v}_{H} \in X_{H}} \frac{\left(\nabla \cdot \mathbf{v}_{H}, q_{H}\right)}{\left\|\mathbf{v}_{H}\right\|_{1} \cdot\left\|q_{H}\right\|_{0}}
$$

for a positive constant $\beta_{*}$. The construction of the finite element spaces $X_{H}$ and $M_{H}$ to satisfy (1.5) can be found in [16], examples include the MINI element and the TaylorHood element.

From the computational point of view, it is convenient to use the same interpolation of the velocity and pressure. However, this choice turns out to violate condition (1.5); therefore, some stabilized methods have been proposed to approximate problem (1.2). Examples of these stabilized methods are those of Brezzi and Douglas [4], Brezzi and Pitkäanta [5], Douglas and Wang [12] (see also the references therein). Recently, a novel stabilized technique using polynomial pressure projection has been proposed and studied to solve incompressible flow $[2,21,22,24]$. This new stabilized method has three prominent features. (1) It is of practical convenience in real applications with the same partitions for velocity and pressure. (2) Less computational time is required by easily applying the lower order elements. (3) Compared with the standard finite element method, its analyses of $H^{1}$-norm and $L^{2}$-norm for the velocity and pressure are derived without any high order regularity assumptions on the exact solution. So this method has been widely used to consider various kinds of problems [17, 18, 25, 28, 36].

In this paper, based on the regular triangular partitions of domain $\Omega$ and the pressure project method, we consider the stabilized adaptive finite volume method for the Oseen equations by the lowest equal order element (i.e., $P_{1}-P_{1}$ pair). The main contributions of our work can be listed as follows.

(I) Existence and uniqueness of stabilized finite element and finite volume schemes are developed, the corresponding stability and convergence results can be established following the references [3, 6, 21].

(II) Residual type a posteriori error estimators are designed, the upper and lower bounds are presented, a discrete local lower bound between two successive numerical solutions is also shown.

(III) Thanks to the local error estimator, the stabilized adaptive finite volume method and its convergence analysis are given and analyzed. 
The outline of this paper is organized as follows. In Sect. 2, the stabilized finite element and finite volume methods for the Oseen problem are described. Our numerical schemes are based on the lowest equal order pair, and we use the polynomial pressure projection method to overcome the restriction of (1.5). The basic idea is to add some terms in the discrete schemes, which are formed by the local projection from different spaces. Having stated the stabilized numerical schemes, we deduce a complete numerical analysis of global upper and local lower bounds for the errors in Sect. 3. Sections 4 and 5 are devoted to deriving the discrete local lower bound and the convergence property of the adaptive stabilized finite volume method. Two numerical examples are presented in Sect. 6 to show the performances of the developed error estimators. One with known solution to verify the convergence orders of the numerical solutions, the other is a model problem to confirm the efficiency of the adaptive finite volume method. Finally, some conclusions are drawn.

\section{Description of the discrete numerical schemes}

\subsection{Stabilized finite element method}

Throughout this paper, we focus on the following finite element subspaces:

$$
\begin{aligned}
& X_{H}=\left\{\mathbf{v} \in C^{0}(\bar{\Omega})^{2} \cap X:\left.\mathbf{v}\right|_{K} \in P_{1}(K)^{2}, \forall K \in \mathcal{T}_{H}\right\}, \\
& M_{H}=\left\{q \in C^{0}(\bar{\Omega}) \cap M:\left.q\right|_{K} \in P_{1}(K), \forall K \in \mathcal{T}_{H}\right\},
\end{aligned}
$$

where $P_{1}(K)$ is the space of affine polynomials on the element $K$.

For the solenoidal vector a, we make the following assumption (see $[3,6,11])$.

Assumption A1 There is a constant $C_{D}$ such that the $m$ derivatives of a within the element $K$ are bounded above by $C_{D}|\mathbf{a}|_{\infty, K}, \forall K \in \mathcal{T}_{h}$.

Under the assumption of weakly divergence free of $\mathbf{a}$, for all $\mathbf{v} \in X$, we have

$$
(\mathbf{a} \cdot \nabla \mathbf{v}, \mathbf{v})=\frac{1}{2}(\mathbf{a} \cdot \nabla(\mathbf{v} \cdot \mathbf{v}), 1)=-\frac{1}{2}(\nabla \cdot \mathbf{a}, \mathbf{v} \cdot \mathbf{v})=0 .
$$

It is well known that the above chosen finite element spaces $X_{H}$ and $M_{H}$ do not satisfy the discrete inf-sup condition (1.5), but they are of practical importance in real applications. A recently popular stabilized approach, called local pressure projection method, is used in $[2,17,21,22]$ to stabilize the lower order finite element for incompressible flow.

The stabilized finite element method for problem (1.2) is to find $\left(\mathbf{u}_{H}, p_{H}\right) \in X_{H} \times M_{H}$ satisfying

$$
\begin{cases}v\left(\nabla \mathbf{u}_{H}, \nabla \mathbf{v}_{H}\right)-\left(\nabla \cdot \mathbf{v}_{H}, p_{H}\right)+\left(\mathbf{a} \cdot \nabla \mathbf{u}_{H}, \mathbf{v}_{H}\right)=\left(\mathbf{f}, \mathbf{v}_{H}\right), & \forall \mathbf{v}_{H} \in X_{H}, \\ \left(\nabla \cdot \mathbf{u}_{H}, q_{H}\right)+G_{h}\left(p_{H}, q_{H}\right)=0, & \forall q_{H} \in M_{H} .\end{cases}
$$

Here, the stabilized term $G(\cdot, \cdot)$ is defined by

$$
G_{h}\left(p_{H}, q_{H}\right)=\left(p_{H}-\Pi_{H} p_{H}, q_{H}-\Pi_{H} q_{H}\right) \quad \forall p_{H}, q_{H} \in M_{H},
$$


and the local projection $\Pi_{H}: L^{2}(\Omega) \rightarrow P_{0}(K)$ satisfies

$$
\begin{aligned}
& \left(p, q_{H}\right)=\left(\Pi_{H} p, q_{H}\right),\left\|\Pi_{H} p\right\|_{0} \leq C\|p\|_{0}, \quad \forall p \in M, q_{H} \in P_{0}(K), \\
& \left\|p-\Pi_{H} p\right\|_{0} \leq C H\|p\|_{1}, \quad \forall p \in H^{1}(\Omega) \cap M
\end{aligned}
$$

where $P_{0}(K)$ denotes a piecewise constant on each element $K$.

We first present the Scott-Zhang [32] interpolating property as the following lemma.

Lemma 2.1 Let $I_{H}$ be the interpolation operator from $X \cap C^{0}(\bar{\Omega})^{2}$ into $X_{h}$. It holds

$$
\begin{aligned}
& \left\|\mathbf{w}-I_{H} \mathbf{w}\right\|_{0, K} \leq C_{1} H_{K}\|\mathbf{w}\|_{1, \omega_{K}}, \\
& \left\|\mathbf{w}-I_{H} \mathbf{w}\right\|_{0, E} \leq C_{1} H_{E}^{1 / 2}\|\mathbf{w}\|_{1, \omega_{E}}, \\
& \left\|I_{H} \mathbf{w}\right\|_{1} \leq\|\mathbf{w}\|_{1},
\end{aligned}
$$

where $\omega_{K}=\bigcup_{K^{\prime} \cap K \neq \emptyset} K^{\prime}\left(K^{\prime} \in \mathcal{T}_{h}\right)$ and $\omega_{E}=\bigcup_{E \cap K \neq \emptyset} K$.

Due to the quasi-uniformness of the triangulation $\mathcal{T}_{H}$, the inverse inequality holds

$$
\left\|\mathbf{w}_{H}\right\|_{1} \leq C_{2} H^{-1}\left\|\mathbf{w}_{H}\right\|_{0}, \quad \forall \mathbf{w}_{H} \in X_{H} .
$$

Denote

$$
\begin{aligned}
& \mathcal{B}\left(\left(\mathbf{u}_{H}, p_{H}\right),\left(\mathbf{v}_{H}, q_{H}\right)\right) \\
& \quad \equiv v\left(\nabla \mathbf{u}_{H}, \nabla \mathbf{v}_{H}\right)-\left(\nabla \cdot \mathbf{v}_{H}, p_{H}\right)+\left(\mathbf{a} \cdot \nabla \mathbf{u}_{H}, \mathbf{v}_{H}\right)+\left(\nabla \cdot \mathbf{u}_{H}, q_{H}\right)+G_{h}\left(p_{H}, q_{H}\right) .
\end{aligned}
$$

Then the well-posedness of problem (2.2) can be obtained from the following theorem.

Theorem 2.2 For all $\left(\mathbf{u}_{H}, p_{H}\right),\left(\mathbf{v}_{H}, q_{H}\right) \in X_{H} \times M_{H}$, it holds

$$
\mathscr{B}\left(\left(\mathbf{u}_{H}, p_{H}\right),\left(\mathbf{v}_{H}, q_{H}\right)\right) \leq C\left(\left\|\mathbf{u}_{H}\right\|_{1}+\left\|p_{H}\right\|_{0}\right)\left(\left\|\mathbf{v}_{H}\right\|_{1}+\left\|q_{H}\right\|_{0}\right) .
$$

Furthermore, there exists a constant $\beta^{*}$ such that, for all $\left(\mathbf{u}_{H}, p_{H}\right) \in X_{H} \times M_{H}$,

$$
\beta^{*}\left(\left\|\nabla \mathbf{u}_{H}\right\|_{0}+\left\|p_{H}\right\|_{0}\right) \leq \sup _{0 \neq\left(\mathbf{v}_{H}, q_{H}\right) \in X_{H} \times M_{H}} \frac{\left|\mathcal{B}\left(\left(\mathbf{u}_{H}, p_{H}\right),\left(\mathbf{v}_{H}, q_{H}\right)\right)\right|}{\left\|\nabla \mathbf{v}_{H}\right\|_{0}+\left\|q_{H}\right\|_{0}} .
$$

Proof The continuity (2.6) holds by using the Cauchy inequality and Assumption A1.

Now, we present the proof of (2.7). For each $p_{H} \in M_{H} \subset M$, there exists $\mathbf{w} \in X[10,16]$ such that

$$
\nabla \cdot \mathbf{w}=p_{H}
$$

and

$$
\|\mathbf{w}\|_{1} \leq C_{3}\left\|p_{H}\right\|_{0}, \quad\left(\nabla \cdot \mathbf{w}, p_{H}\right) \geq C_{4}\|\mathbf{w}\|_{1}\left\|p_{H}\right\|_{0} .
$$


Let $\mathbf{w}_{H}=I_{H} \mathbf{w} \in X_{H}$, which satisfies Lemma 2.1. Then it follows from the Green's formula, Poincare's inequality, the inverse inequality (2.5), and (2.8)-(2.9) that

$$
\begin{aligned}
\left|v\left(\nabla \mathbf{u}_{H}, \nabla \mathbf{w}_{H}\right)\right| & \leq v\left\|\mathbf{u}_{H}\right\|_{1}\left\|\mathbf{w}_{H}\right\|_{1} \\
& \leq v\left\|\mathbf{u}_{H}\right\|_{1}\|\mathbf{w}\|_{1} \leq C_{3} v\left\|\mathbf{u}_{H}\right\|_{1}\left\|p_{H}\right\|_{0} \leq \frac{1}{4}\left\|p_{H}\right\|_{0}^{2}+C_{3}^{2} v^{2}\left\|\mathbf{u}_{H}\right\|_{1}^{2}, \\
\left|\left(\nabla \cdot \mathbf{w}_{H}, p_{H}\right)\right| & =\left(\nabla \cdot \mathbf{w}, p_{H}\right)-\left(\nabla \cdot\left(\mathbf{w}-\mathbf{w}_{H}\right), p_{H}\right) \geq\left\|p_{H}\right\|_{0}^{2}-C_{1} C_{4} H\|\mathbf{w}\|_{1}\left\|\nabla p_{H}\right\|_{0} \\
& =\left\|p_{H}\right\|_{0}^{2}-C_{1} C_{4} H\|\mathbf{w}\|_{1} \sum_{K \in \mathcal{T}_{H}}\left\|\nabla\left(p_{H}-\Pi_{H} p_{H}\right)\right\|_{0} \\
\geq & \left\|p_{H}\right\|_{0}^{2}-C_{1} C_{2} C_{3} C_{4}\left\|p_{H}\right\|_{0} \sum_{K \in \mathcal{T}_{H}}\left\|p_{H}-\Pi_{H} p_{H}\right\|_{0} \\
\geq & \frac{3}{4}\left\|p_{H}\right\|_{0}^{2}-C_{1}^{2} C_{2}^{2} C_{3}^{2} C_{4}^{2}\left\|p_{H}-\Pi_{H} p_{H}\right\|_{0}^{2},
\end{aligned}
$$

$\left|\left(\mathbf{a} \cdot \nabla \mathbf{u}_{H}, \mathbf{w}_{H}\right)\right| \leq C_{D}|\mathbf{a}|_{\infty}\left\|\nabla \mathbf{u}_{H}\right\|_{0}\left\|\mathbf{w}_{H}\right\|_{0}$

$$
\leq C_{D}|\mathbf{a}|_{\infty}\left\|\nabla \mathbf{u}_{H}\right\|_{0}\left\|\mathbf{w}_{H}\right\|_{1} \leq \frac{1}{4}\left\|p_{H}\right\|_{0}^{2}+C_{3}^{2} C_{D}^{2}|\mathbf{a}|_{\infty}^{2}\left\|\mathbf{u}_{H}\right\|_{1}^{2} .
$$

Set $\delta>0$ and take

$$
\mathbf{v}_{H}=\mathbf{u}_{H}-\delta \mathbf{w}_{H} \quad \text { and } \quad q_{H}=p_{H} .
$$

Thanks to (2.1) and the above inequalities, one finds

$$
\begin{aligned}
&\left|\mathcal{B}\left(\left(\mathbf{u}_{H}, p_{H}\right),\left(\mathbf{u}_{H}-\delta \mathbf{w}_{H}, p_{H}\right)\right)\right| \\
&=\mid v\left(\nabla \mathbf{u}_{H}, \nabla \mathbf{u}_{H}\right)+G_{h}\left(p_{H}, p_{H}\right)+\left(\mathbf{a} \cdot \nabla \mathbf{u}_{H}, \mathbf{u}_{H}\right) \\
&-\delta\left[v\left(\nabla \mathbf{u}_{H}, \nabla \mathbf{w}_{H}\right)-\left(\nabla \cdot \mathbf{w}_{H}, p_{H}\right)+\left(\mathbf{a} \cdot \nabla \mathbf{u}_{H}, \mathbf{w}_{H}\right)\right] \mid \\
& \geq v\left\|\nabla \mathbf{u}_{H}\right\|_{0}^{2}+\left\|\left(I-\Pi_{H}\right) p_{H}\right\|_{0}^{2} \\
&-\delta\left(\left(C_{3}^{2} v^{2}+C_{3}^{2} C_{D}^{2}|\mathbf{a}|_{\infty}^{2}\right)\left\|\mathbf{u}_{H}\right\|_{1}^{2}-\frac{1}{4}\left\|p_{H}\right\|_{0}^{2}+C_{1}^{2} C_{2}^{2} C_{3}^{2} C_{4}^{2}\left\|p_{H}-\Pi_{H} p_{H}\right\|_{0}^{2}\right) \\
& \geq\left(v-\delta\left(C_{3}^{2} v^{2}+C_{3}^{2} C_{D}^{2}|\mathbf{a}|_{\infty}^{2}\right)\right)\left\|\mathbf{u}_{H}\right\|_{1}^{2}+\frac{\delta}{4}\left\|p_{H}\right\|_{0}^{2}+\left(1-\delta C_{1}^{2} C_{2}^{2} C_{3}^{2} C_{4}^{2}\right)\left\|p_{H}-\Pi_{H} p_{H}\right\|_{0}^{2},
\end{aligned}
$$

provided that $0<\delta<\min \left\{\frac{v}{C_{3}^{2}\left(v^{2}+C_{D}^{2}|\mathbf{a}|_{\infty}^{2}\right)}, \frac{1}{C_{1}^{2} C_{2}^{2} C_{3}^{2} C_{4}^{2}}\right\}$. Denote

$$
C(v) \equiv \min \left\{v-\delta C_{3}^{2}\left(v^{2}+C_{D}^{2}|\mathbf{a}|_{\infty}^{2}\right), \frac{1}{4} \delta\right\}, \quad C(\delta) \equiv \max \left\{2,1+2 \delta^{2}\right\} .
$$

Then we have

$$
\begin{aligned}
\left\|\nabla \mathbf{v}_{H}\right\|_{0}^{2}+\left\|q_{H}\right\|_{0}^{2} & =\left\|\nabla \mathbf{u}_{H}-\delta \nabla \mathbf{w}_{H}\right\|_{0}^{2}+\left\|p_{H}\right\|_{0}^{2} \\
& \leq 2\left\|\nabla \mathbf{u}_{H}\right\|_{0}^{2}+\left(1+2 \delta^{2}\right)\left\|p_{H}\right\|_{0}^{2} \leq C(\delta)\left(\left\|\nabla \mathbf{u}_{H}\right\|_{0}^{2}+\left\|p_{H}\right\|_{0}^{2}\right) .
\end{aligned}
$$

Taking $\beta^{*}=C(v) / C(\delta)$, we obtain the desired result (2.7).

From Theorem 2.2 we see that problem (2.2) admits a unique solution. 


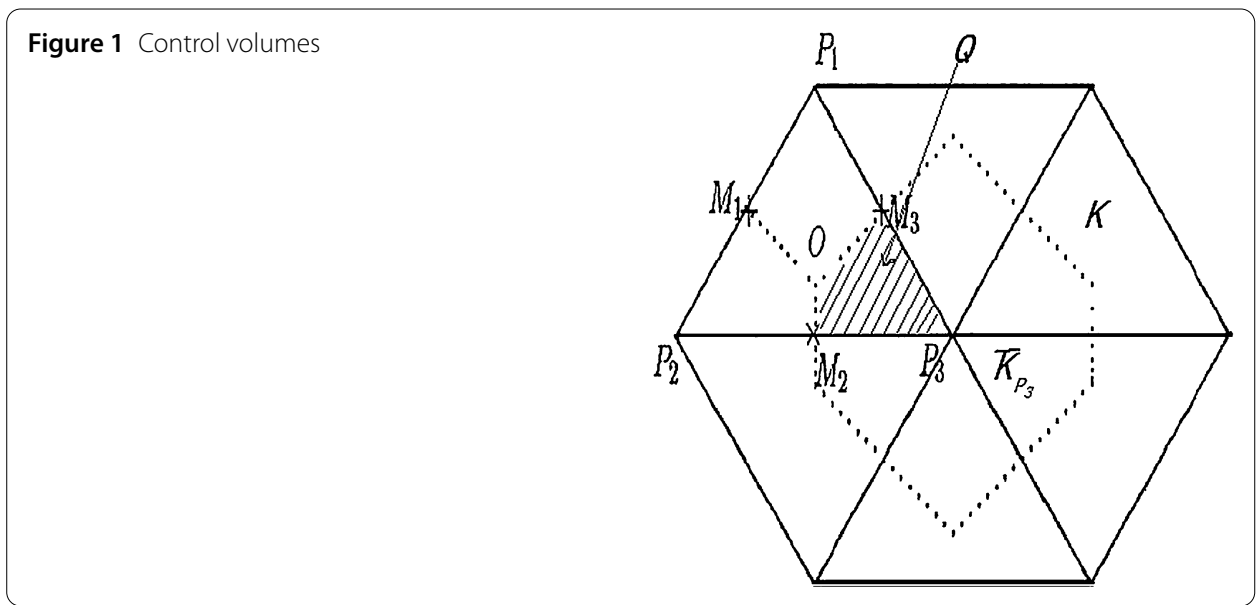

\subsection{Stabilized finite volume method}

Let $\mathcal{N}_{H}$ be the set of all interior vertices of the triangulation and $\mathcal{E}_{H}$ be the set of all interior edges. To define the finite volume method, we introduce a dual partition $\mathcal{T}_{H}^{*}$ based on $\mathcal{T}_{H}$, the elements in $\mathcal{T}_{H}^{*}$ are called control volumes. The dual mesh is constructed by the following rule: For each element $K \in \mathcal{T}_{H}$ with vertices $P_{j}, j=1,2,3$, select its barycenter $O$ and the midpoint $M_{j}$ on each of the edges of $K$. We can construct the control volumes $\widetilde{K}_{i} \in \mathcal{T}_{H}^{*}$ by connecting $O$ to $M_{j}$ as shown in Fig. 1 .

The dual finite element space is defined by

$$
\widetilde{X}_{H}=\left\{\mathbf{v} \in L^{2}(\Omega)^{2}:\left.\mathbf{v}\right|_{\widetilde{K}_{i}} \in P_{0}\left(\widetilde{K}_{i}\right)^{2},\left.\mathbf{v}\right|_{\partial \widetilde{K}_{i} \cap \partial \Omega}=0, \forall \widetilde{K}_{i} \in \mathcal{T}_{H}^{*}\right\}
$$

Note that $P_{0}\left(\widetilde{K}_{i}\right)$ denotes a piecewise constant on each control volume $\widetilde{K}_{i}$. The two finite dimensional spaces $X_{H}$ and $\widetilde{X}_{H}$ have the same dimension. Furthermore, there exists an invertible linear mapping $\Gamma_{H}: X_{H} \rightarrow \widetilde{X}_{H}$ such that

$$
\Gamma_{H} \mathbf{v}_{H}(x)=\sum_{i=1}^{\mathcal{N}_{H}} \mathbf{v}_{H}\left(x_{i}\right) \phi_{i}(x), x_{i} \quad \text { is the node of } \mathcal{T}_{H}, x \in \Omega, v_{H} \in X_{H},
$$

where $\phi_{i}(x)$ is the characteristic function associated with the dual partition $\mathcal{T}_{H}^{*}$ :

$$
\phi_{i}(x)= \begin{cases}1 & x \in \widetilde{K}_{i} \\ 0 & \text { otherwise }\end{cases}
$$

The idea of connecting the different spaces through the mapping $\Gamma_{H}$ was introduced by $\mathrm{Li}$ and Zhu in [27] for the elliptic problem. The following properties hold.

Lemma 2.3 (See $[7,26,34])$ For any $\mathbf{v}_{H} \in X_{H}$ and $\mathbf{v}_{H}^{*}=\Gamma_{H} \mathbf{v}_{H} \in \widetilde{X}_{H}$, for each interior element $K \in \mathcal{T}_{H}$ with its boundary $\partial K \in \mathcal{E}_{H}$, there hold

$$
\begin{aligned}
& \int_{K}\left(\mathbf{v}_{H}-\mathbf{v}_{H}^{*}\right) d x=0, \quad \int_{\partial K}\left(\mathbf{v}_{H}-\mathbf{v}_{H}^{*}\right) d s=0, \quad\left\|\mathbf{v}_{H}^{*}\right\|_{0} \leq C\left\|v_{H}\right\|_{0}, \\
& \left\|\mathbf{v}_{H}-\mathbf{v}_{H}^{*}\right\|_{0, r, K} \leq C h_{K}\left\|v_{H}\right\|_{1, r, K}, \quad\left\|\mathbf{v}_{H}-\mathbf{v}_{H}^{*}\right\|_{0, r, \partial K} \leq C H_{K}^{1-1 / r}\left\|\mathbf{v}_{h}\right\|_{1, r, K}, \quad r \in[1, \infty) .
\end{aligned}
$$


Analogous to (2.2), the stabilized finite volume method for problem (1.1) is to find $\left(\mathbf{u}_{H}^{v}, p_{H}^{v}\right) \in\left(X_{H}, M_{H}\right)$ satisfying

$$
\begin{cases}A\left(\mathbf{u}_{H}^{v}, \Gamma_{H} \mathbf{v}_{H}\right)+D\left(\Gamma_{H} \mathbf{v}_{H}, p_{H}^{v}\right)+\left(\mathbf{a} \cdot \nabla \mathbf{u}_{H}^{v}, \Gamma_{H} \mathbf{v}_{H}\right)=\left(\mathbf{f}, \Gamma_{h} \mathbf{v}_{H}\right), & \forall \mathbf{v}_{H} \in X_{H}, \\ \left(\nabla \cdot \mathbf{u}_{H}^{v}, q_{H}\right)+G_{H}\left(p_{H}^{v}, q_{H}\right)=0, & \forall q_{H} \in M_{H},\end{cases}
$$

where

$$
\begin{aligned}
& A\left(\mathbf{u}_{H}^{v}, \Gamma_{h} \mathbf{v}_{H}\right)=-v \sum_{j=1}^{\mathcal{N}_{H}} \mathbf{v}_{H}\left(P_{j}\right) \cdot \int_{\partial \widetilde{K}_{j}} \frac{\partial \mathbf{u}_{H}^{v}}{\partial \mathbf{n}} d s, \\
& D\left(\Gamma_{H} \mathbf{v}_{H}, p_{H}^{v}\right)=\sum_{j=1}^{\mathcal{N}_{H}} \mathbf{v}_{H}\left(P_{j}\right) \cdot \int_{\partial \widetilde{K}_{j}} p_{H}^{v} \cdot \mathbf{n} d s \\
& \left(\mathbf{f}, \Gamma_{H} \mathbf{v}_{H}\right)=\sum_{j=1}^{\mathcal{N}_{H}} \mathbf{v}_{H}\left(P_{j}\right) \cdot \int_{\widetilde{K}_{j}} \mathbf{f} d x .
\end{aligned}
$$

Lemma 2.4 It holds that, for all $\mathbf{u}_{H}^{v}, \mathbf{v}_{H} \in X_{H}, p_{H}^{v} \in M_{H}$,

$$
\begin{aligned}
& A\left(\mathbf{u}_{H}^{v}, \Gamma_{H} \mathbf{v}_{H}\right)=v\left(\nabla \mathbf{u}_{H}^{v}, \nabla \mathbf{v}_{H}\right), \\
& D\left(\Gamma_{H} \mathbf{v}_{H}, p_{H}^{v}\right)=\left(\nabla \cdot \mathbf{v}_{H}, p_{H}^{v}\right) .
\end{aligned}
$$

Moreover, we have

$$
\left(\mathbf{a} \cdot \nabla \mathbf{u}_{H}^{v}, \Gamma \mathbf{u}_{H}^{v}\right)=0
$$

Proof The equations in (2.11) have been shown in [23, 34, 35]. It suffices to prove (2.12). For $\mathbf{u}_{H}^{v} \in X_{H}$, it follows from the definition of $\Gamma_{h}$ and (2.1) that

$$
\begin{aligned}
\left(\mathbf{a} \cdot \nabla \mathbf{u}_{H}^{v}, \Gamma \mathbf{u}_{H}^{v}\right) & =\sum_{j=1}^{N_{H}} \int_{\widetilde{K}_{j}} \mathbf{a} \cdot \nabla \mathbf{u}_{H}^{v} \cdot \Gamma_{H} \mathbf{u}_{H}^{v} d x \\
& =-\sum_{j=1}^{N_{H}} \Gamma_{H} \mathbf{u}_{H}^{v}\left(P_{j}\right) \cdot \int_{\widetilde{K}_{j}} \mathbf{a} \cdot \nabla \mathbf{u}_{H}^{v} d x \\
& =\sum_{j=1}^{N_{H}} \Gamma_{H} \mathbf{u}_{H}^{v}\left(P_{j}\right) \cdot\left[\int_{\widetilde{K}_{j}}(\nabla \cdot \mathbf{u}) u_{H}^{v} d x-\int_{\partial \widetilde{K}_{j}} \mathbf{a u}_{H}^{v} \cdot \mathbf{n} d s\right] .
\end{aligned}
$$

With the weakly divergence free of $\mathbf{a}$ and the continuity of $\mathbf{a}, \mathbf{u}_{H}^{v}$, we complete the proof.

We denote the generalized bilinear form $\mathcal{C}((\cdot, \cdot),(\cdot, \cdot))$ on $\left(X_{H}, M_{H}\right) \times\left(X_{H}, M_{H}\right)$ as

$$
\begin{aligned}
& \mathcal{C}\left(\left(\mathbf{u}_{H}^{v}, p_{H}^{v}\right),\left(\mathbf{v}_{H}, q_{H}\right)\right) \\
& \quad=A\left(\mathbf{u}_{H}^{v}, \Gamma_{H} \mathbf{v}_{H}\right)+D\left(\Gamma_{H} \mathbf{v}_{H}, p_{H}^{v}\right)+d\left(\mathbf{u}_{H}^{v}, q_{H}\right)+G_{H}\left(p_{H}^{v}, q_{H}\right)+\left(\mathbf{a} \cdot \nabla \mathbf{u}_{H}^{v}, \Gamma_{H} \mathbf{v}_{H}\right) .
\end{aligned}
$$


By applying the relationships between finite element and finite volume methods presented in Lemma 2.4, and following the proof of Theorem 2.2, we can establish the continuity and weak coercivity for the generalized bilinear form $\mathcal{C}((\cdot, \cdot),(\cdot, \cdot))$. Here we omit the proof for simplification and present its continuity and weak coercivity.

Theorem 2.5 For all $\left(\mathbf{u}_{H}^{v}, p_{H}^{v}\right),\left(\mathbf{v}_{H}, q_{H}\right) \in X_{H} \times M_{H}$, it holds

$$
\left|\mathcal{C}\left(\left(\mathbf{u}_{H}^{v}, p_{H}^{v}\right),\left(\mathbf{v}_{H}, q_{H}\right)\right)\right| \leq C\left(\left\|\mathbf{u}_{H}^{v}\right\|_{1}+\left\|p_{H}^{v}\right\|_{0}\right)\left(\left\|\mathbf{v}_{H}\right\|_{1}+\left\|q_{H}\right\|_{0}\right) .
$$

Moreover, there exists a constant $\widetilde{\beta}^{*}>0$, independent of $H$, such that

$$
\widetilde{\beta}^{*}\left(\left\|\mathbf{u}_{H}^{v}\right\|_{1}+\left\|p_{H}^{v}\right\|_{0}\right) \leq \sup _{0 \neq\left(\mathbf{v}_{H}, q_{H}\right) \in\left(X_{H}, M_{H}\right)} \frac{\mathcal{C}\left(\left(\mathbf{u}_{H}^{v}, p_{H}^{v}\right),\left(\mathbf{v}_{H}, q_{H}\right)\right)}{\left\|\mathbf{v}_{H}\right\|_{1}+\left\|q_{H}\right\|_{0}} .
$$

From Theorem 2.5, we know that problem (2.10) has a unique solution $\left(\mathbf{u}_{H}^{v}, p_{H}^{v}\right)$.

For the stability and convergence results of numerical schemes (2.2) and (2.10), following the proofs provided in $[3,6,21]$, by taking different test functions and using the energy method, we can obtain that the numerical schemes (2.2) and (2.10) are unconditionally stable, error estimates for the numerical solutions are also optimal. Here we omit these proofs for simplification.

\section{A posteriori error estimation}

In this section, we derive a residual type error estimator of the stabilized finite volume method for the Oseen problem. The upper and lower bounds between the exact solution and the finite volume solution are obtained by using Lemma 2.4 and some techniques involving the bubble functions.

\subsection{Upper bounds}

In this subsection, a residual-based error estimator is investigated by using the techniques of residual a posteriori error estimates and the stabilized finite volume method for the Oseen equations. The error between $(\mathbf{u}, p)$ and $\left(\mathbf{u}_{H}^{v}, p_{H}^{v}\right)$ is bounded by the global error estimator $\eta_{H}$ defined below.

For an element $K$ of the triangulation $\mathcal{T}_{H}$, we set

$$
R_{K}=\mathbf{f}+v \Delta \mathbf{u}_{H}^{v}-\nabla p_{H}^{v}-\mathbf{a} \cdot \nabla \mathbf{u}_{H}^{v},
$$

and for an edge $E$ of a triangle $K$

$$
J_{E}=\left[v \frac{\partial \mathbf{u}_{H}^{v}}{\partial \mathbf{n}}-p_{H}^{v} \mathbf{I} \cdot \mathbf{n}\right]_{E}=\left[v \frac{\partial \mathbf{u}_{H}^{v}}{\partial \mathbf{n}}\right]_{E},
$$

where $\mathbf{n}$ is the unit normal outward to $\partial K$. We also define the following local error estimator for any $\left(\mathbf{u}_{H}^{v}, p_{H}^{v}\right) \in X_{H} \times M_{H}$ :

$$
\eta_{H}^{2}(K)=H_{K}^{2}\left\|R_{K}\right\|_{0, K}^{2}+\left\|\nabla \cdot \mathbf{u}_{H}^{v}\right\|_{0, K}^{2}+H_{E}\left\|J_{E}\right\|_{0, E}^{2} .
$$

Then the global error estimator is given by

$$
\eta_{H}^{2}=\sum_{K \in \mathcal{T}_{H}} \eta_{H}^{2}(K)
$$


The following theorem plays an important role in the process of establishing the upper bound of adaptive finite volume method for the Oseen equations.

Theorem 3.1 Let $(\mathbf{u}, p)$ and $\left(\mathbf{u}_{H}^{v}, p_{H}^{v}\right)$ be the solutions of problems (1.2) and (2.10), respectively. Then, for any $(\mathbf{v}, q) \in X \times M$ and $\mathbf{v}_{H}^{*} \in \widetilde{X}_{H}$, it holds

$$
\begin{gathered}
v\left(\nabla\left(\mathbf{u}-\mathbf{u}_{H}^{v}\right), \nabla \mathbf{v}\right)-\left(\nabla \cdot \mathbf{v}, p-p_{H}^{v}\right)+\left(\nabla \cdot\left(\mathbf{u}-\mathbf{u}_{H}^{v}\right), q\right)+\left(\mathbf{a} \cdot \nabla\left(\mathbf{u}-\mathbf{u}_{H}^{v}\right), \mathbf{v}\right) \\
\quad=\sum_{K \in \mathcal{T}_{H}} \int_{K}\left(R_{K} \cdot\left(\mathbf{v}-\mathbf{v}_{H}^{*}\right)+\nabla \cdot \mathbf{u}_{H}^{v} q\right) d x+\sum_{E \in \mathcal{E}_{H}} \int_{E} J_{E}\left(\mathbf{v}-\mathbf{v}_{H}^{*}\right) d s .
\end{gathered}
$$

Proof It follows from (1.2) that

$$
\begin{aligned}
& v\left(\nabla\left(\mathbf{u}-\mathbf{u}_{H}^{v}\right), \nabla \mathbf{v}\right)-\left(\nabla \cdot \mathbf{v}, p-p_{H}^{v}\right)+\left(\nabla \cdot\left(\mathbf{u}-\mathbf{u}_{H}^{v}\right), q\right) \\
& \quad=(\mathbf{f}, \mathbf{v})-(\mathbf{a} \cdot \nabla \mathbf{u}, \mathbf{v})-v\left(\nabla \mathbf{u}_{H}^{v}, \nabla \mathbf{v}\right)+\left(\nabla \cdot \mathbf{v}, p_{H}^{v}\right)-\left(\nabla \cdot \mathbf{u}_{H}^{v}, q\right) .
\end{aligned}
$$

By the Green's formula, one finds

$$
\begin{aligned}
& v\left(\nabla \mathbf{u}_{H}^{v}, \nabla \mathbf{v}\right)-\left(\nabla \cdot \mathbf{v}, p_{H}^{v}\right)+\left(\nabla \cdot \mathbf{u}_{H}^{v}, q\right) \\
& \quad=\left(-v \Delta \mathbf{u}_{H}^{v}+\nabla p_{H}^{v}, \mathbf{v}\right)+\left(\nabla \cdot \mathbf{u}_{H}^{v}, q\right)+\sum_{E \in \mathcal{E}_{H}} \int_{E} J_{E} \cdot \mathbf{v} d s .
\end{aligned}
$$

On each control volume $\widetilde{K} \in \mathcal{T}_{H}^{*}$, applying the Green's formula on $\widetilde{K} \cap K$ (see Fig. 1), we have

$$
\int_{\widetilde{K} \cap K}\left(-v \Delta \mathbf{u}_{H}^{v}+\nabla p_{H}^{v}\right) \cdot \mathbf{v}_{H}^{*} d x=-\int_{\partial \widetilde{K} \cap K} J_{E} \cdot \mathbf{v}_{H}^{*} d s-\int_{\partial K \cap \widetilde{K}} J_{E} \cdot \mathbf{v}_{H}^{*} d s .
$$

Summing the above results and using the first equation in (2.10), we obtain

$$
\begin{aligned}
0= & \left(-v \Delta \mathbf{u}_{H}^{v}+\nabla p_{H}^{v}, \mathbf{v}_{H}^{*}\right)+\left(\mathbf{f}, \mathbf{v}_{H}^{*}\right)-\left(\mathbf{a} \cdot \nabla \mathbf{u}_{H}^{v}, \mathbf{v}_{H}^{*}\right)+\sum_{E \in \mathcal{E}_{H}} \int_{E} J_{E} \cdot \mathbf{v}_{H}^{*} d s \\
= & \left(-v \Delta \mathbf{u}_{H}^{v}+\nabla p_{H}^{v}, \mathbf{v}_{H}^{*}\right)+\left(\mathbf{f}, \mathbf{v}_{H}^{*}\right)+\left(\mathbf{a} \cdot \nabla \mathbf{u}_{H}^{v}, \mathbf{u}-\mathbf{v}_{H}^{*}\right) \\
& +\sum_{E \in \mathcal{E}_{H}} \int_{E} J_{E} \cdot \mathbf{v}_{H}^{*} d s-\left(\mathbf{a} \cdot \nabla \mathbf{u}_{H}^{v}, \mathbf{v}\right) .
\end{aligned}
$$

The proof is completed by using (3.2), (3.3), and (3.4).

Theorem 3.2 Let $(\mathbf{u}, p)$ and $\left(\mathbf{u}_{H}^{v}, p_{H}^{v}\right)$ be the solutions of problems (1.2) and (2.10), respectively. Under Assumption A1, there exists a positive constant $C$ such that

$$
\left\|\nabla\left(\mathbf{u}-\mathbf{u}_{H}^{v}\right)\right\|_{0}+\left\|p-p_{H}^{v}\right\|_{0} \leq C_{5} \eta_{H} .
$$

Proof Using Lemmas 2.1 and 2.3 and the Cauchy-Schwarz inequality in (3.1), we see that

$$
\left|\sum_{K \in \mathcal{T}_{H}} \int_{K} R_{K} \cdot\left(\mathbf{v}-I_{H} \mathbf{v}\right) d x\right| \leq C_{1}\left(\sum_{K \in \mathcal{T}_{h}} H_{K}^{2}\left\|R_{K}\right\|_{0, K}^{2}\right)^{1 / 2}\|\mathbf{v}\|_{1},
$$




$$
\begin{aligned}
& \left|\sum_{K \in \mathcal{T}_{H}} \int_{K} \nabla \cdot \mathbf{u}_{H}^{v} \cdot q d x\right| \leq \sum_{K \in \mathcal{T}_{H}}\left\|\nabla \cdot u_{H}^{v}\right\|_{0, K} \cdot\|q\|_{0}, \\
& \left|\sum_{E \in \mathcal{E}_{H}} \int_{E} J_{E} \cdot\left(\mathbf{v}-I_{H} \mathbf{v}\right) d s\right| \leq C_{1}\left(\sum_{E \in \mathcal{E}_{h}} H_{E}\left\|J_{E}\right\|_{0, E}^{2}\right)^{1 / 2}\|\mathbf{v}\|_{1} .
\end{aligned}
$$

It follows from the above inequalities, Lemma 2.3, and the coerciveness property (1.3) that

$$
\begin{aligned}
\beta( & \left.\left\|\nabla\left(\mathbf{u}-\mathbf{u}_{H}^{v}\right)\right\|_{0}+\left\|p-p_{H}^{v}\right\|_{0}\right) \\
\leq & \sup _{0 \neq(\mathbf{v}, q) \in X \times M}\left(\mid v\left(\nabla\left(\mathbf{u}-\mathbf{u}_{H}^{v}\right), \nabla \mathbf{v}\right)-\left(\nabla \cdot \mathbf{v}, p-p_{H}^{v}\right)+\left(\mathbf{a} \cdot \nabla\left(\mathbf{u}-\mathbf{u}_{H}^{v}\right), \mathbf{v}\right)\right. \\
& \left.+\left(\nabla \cdot\left(\mathbf{u}-\mathbf{u}_{H}^{v}\right), q\right) \mid\right) /\left(\|\nabla \mathbf{v}\|_{0}+\|q\|_{0}\right) \\
\leq & C_{5}\left(\sum_{K \in \mathcal{T}_{H}} H_{K}^{2}\left\|R_{K}\right\|_{K}^{2}+\sum_{K \in \mathcal{T}_{H}}\left\|\nabla \cdot u_{H}^{v}\right\|_{K}^{2}+\sum_{E \in \mathcal{E}_{H}} H_{E}\left\|J_{E}\right\|_{E}^{2}\right)^{1 / 2} \leq C_{5} \eta_{H} .
\end{aligned}
$$

Thus, we complete the proof.

\subsection{Lower bounds}

This subsection is devoted to estimating the lower bound of the residual based error estimator. Here, it is important to ensure the efficiency of an algorithm that uses $\eta_{H}$ as a local refinement indicator. Firstly, we recall the definition of the oscillation of the residual on each element $K \in \mathcal{T}_{H}$ :

$$
\operatorname{OSC}_{R}^{2}(K) \equiv\left\|H_{K}\left(R_{K}-\bar{R}_{K}\right)\right\|_{K}^{2}, \quad O S C_{D}^{2}(K)=\left\|\nabla \cdot \mathbf{u}_{H}^{v}\right\|_{K}^{2}
$$

where $\bar{R}_{K}$ is defined by $\bar{R}_{K}=\frac{1}{|K|} \int_{K} R d x,|K|$ is the area of an element $K$. Moreover, we define the oscillation of the jump on each edge $E$ by

$$
\operatorname{OSC}_{J}^{2}(E) \equiv H_{E}\left\|J_{E}-\bar{J}_{E}\right\|_{E}^{2}=H_{E}\left\|\left[\frac{\partial \mathbf{u}_{H}^{v}}{\partial \mathbf{n}}-\frac{1}{H_{E}} \int_{E} \frac{\partial \mathbf{u}_{H}^{v}}{\partial \mathbf{n}} d s\right]\right\|_{E}^{2}=0 .
$$

Then the local oscillation is defined by

$$
O S C_{H}(K)=\left(O S C_{R}^{2}(K)+O S C_{D}^{2}(K)+O S C_{J}^{2}(E)\right)^{1 / 2}
$$

and the global oscillation by

$$
\begin{aligned}
\operatorname{OSC}_{H} & =\left(\sum_{K \in \mathcal{T}_{H}}\left(\operatorname{OSC}_{R}^{2}(K)+O S C_{D}^{2}(K)\right)\right)^{\frac{1}{2}} \\
& =\left(\sum_{K \in \mathcal{T}_{H}}\left(\left\|H_{K}\left(R_{K}-\bar{R}_{K}\right)\right\|_{K}^{2}+\left\|\nabla \cdot \mathbf{u}_{H}^{v}\right\|_{K}^{2}\right)\right)^{\frac{1}{2}} .
\end{aligned}
$$

To analyze the residual-based error estimator, the triangular bubble functions are introduced as follows. For a triangle $K \in \mathcal{T}_{H}$, let $\lambda_{K, 1}, \lambda_{K, 2}$, and $\lambda_{K, 3}$ be the barycentric coordi- 
nates of $K$. Define the triangular bubble function $\mathbf{b}_{K}$ by

$$
\mathbf{b}_{K}(K)= \begin{cases}27 \lambda_{K, 1} \lambda_{K, 2} \lambda_{K, 3}, & \text { in } K, \\ 0, & \text { in } \Omega \backslash T .\end{cases}
$$

Also, for any $E \in \mathcal{E}_{H}$, let the barycentric coordinates of the end points of $E$ be $\lambda_{E, 1}$ and $\lambda_{E, 2}$, and define the edge bubble function $b_{E}$ by

$$
b_{E}(K)= \begin{cases}4 \lambda_{E, 1} \lambda_{E, 2}, & \text { in } \omega_{E}, \\ 0, & \text { in } \Omega \backslash \omega_{E},\end{cases}
$$

where $\omega_{E}$ is defined in Lemma 2.1. The following results can be found in reference [33].

Lemma 3.3 Assume that the partition $\mathcal{T}_{H}$ is locally quasi-uniform. For any element $K \in \mathcal{T}_{H}$ and edge $E \in \mathcal{E}_{H}$, the functions $\mathbf{b}_{K}$ and $b_{E}$ satisfy the following properties:

(1) $\operatorname{supp} \mathbf{b}_{K} \subset K, \boldsymbol{b}_{K} \in[0,1]$, and $\max _{x \in K} \mathbf{b}_{K}(x)=1$;

$$
\int_{K} \mathbf{b}_{K} d x=\frac{9}{20}|K| \sim H_{K}^{2}, \quad\left\|\nabla \mathbf{b}_{K}\right\|_{0, K} \leq C H_{K}^{-1}\left\|\mathbf{b}_{K}\right\|_{K} .
$$

(2) $\operatorname{supp} b_{E} \subset \omega_{E}, b_{E} \in[0,1]$, and $\max _{x \in \omega_{E}} b_{E}(x)=1$;

$$
\int_{E} b_{E} d x=\frac{2}{3} H_{E}, \quad \int_{\omega_{E}} b_{E} d x \sim H_{E}^{2}, \quad\left\|\nabla b_{E}\right\|_{\omega_{E}} \leq C H_{E}^{-1}\left\|b_{E}\right\|_{\omega_{E}} .
$$

Lemma 3.4 For any $K \in \mathcal{T}_{H}$ and $\left(\mathbf{u}_{H}^{v}, p_{H}^{v}\right) \in X_{H} \times M_{H}$, under Assumption A1, we have

$$
\begin{aligned}
& \left\|H_{K} R_{K}\right\|_{K} \leq C\left(\left\|\nabla\left(\mathbf{u}-\mathbf{u}_{H}^{v}\right)\right\|_{K}+\left\|p-p_{H}^{v}\right\|_{K}+\left\|H_{K}\left(R_{K}-\bar{R}_{K}\right)\right\|_{K}\right), \\
& \left\|H_{E}^{\frac{1}{2}} J_{E}\right\|_{E} \leq C\left(\left\|\nabla\left(\mathbf{u}-\mathbf{u}_{H}^{v}\right)\right\|_{\omega_{E}}+\left\|p-p_{H}^{v}\right\|_{\omega_{E}}+\left\|H_{K}\left(R_{K}-\bar{R}_{K}\right)\right\|_{\omega_{K}}\right) .
\end{aligned}
$$

Proof It follows from (1.2), (2.1), inverse inequality, the Green's formula, and Lemma 3.3 that

$$
\begin{aligned}
\left\|\bar{R}_{1 K}\right\|_{K}^{2}= & \frac{20}{9}\left(\int_{K} \mathbf{b}_{K} R_{1 K} \bar{R}_{K} d x+\int_{K} \mathbf{b}_{K} \bar{R}_{K}\left(\bar{R}_{K}-R_{K}\right) d x\right)_{K} \\
\approx & \left(\mathbf{f}+v \Delta \mathbf{u}_{H}^{v}-\mathbf{a} \cdot \nabla \mathbf{u}_{H}^{v}-\nabla p_{H}^{v}, \mathbf{b}_{K} \bar{R}_{K}\right)_{K}+\int_{K} \mathbf{b}_{K} \bar{R}_{K}\left(\bar{R}_{K}-R_{K}\right) d x \\
= & \left(v \nabla \mathbf{u}, \nabla\left(\mathbf{b}_{K} \bar{R}_{K}\right)\right)_{K}-\left(p, \nabla \cdot\left(\mathbf{b}_{K} \bar{R}_{K}\right)\right)_{K}+\left(\mathbf{a} \cdot \nabla \mathbf{u}, \mathbf{b}_{K} \bar{R}_{K}\right)_{K} \\
& -\left(v \nabla \mathbf{u}_{H}^{v}, \nabla\left(\mathbf{b}_{K} \bar{R}_{K}\right)\right)_{K} \\
& +\left(p_{H}^{v}, \nabla \cdot\left(\mathbf{b}_{K} \bar{R}_{K}\right)\right)_{K}-\left(\mathbf{a} \cdot \nabla \mathbf{u}_{H}^{v}, \mathbf{b}_{K} \bar{R}_{K}\right)_{K}+\int_{K} \mathbf{b}_{K} \bar{R}_{K}\left(\bar{R}_{K}-R_{K}\right) d x \\
\leq & C\left[H_{K}^{-1}\left(\left\|\nabla\left(\mathbf{u}-\mathbf{u}_{H}^{v}\right)\right\|_{K}+\left\|p-p_{H}^{v}\right\|_{K}\right)+\left\|\bar{R}_{K}-R_{K}\right\|_{K}\right]\left\|\bar{R}_{K}\right\|_{K} .
\end{aligned}
$$

Thus we have

$$
\left\|\bar{R}_{1 K}\right\|_{K} \leq C\left(H_{K}^{-1}\left(\left\|\nabla\left(\mathbf{u}-\mathbf{u}_{H}^{v}\right)\right\|_{K}+\left\|p-p_{H}^{v}\right\|_{K}\right)+\left\|\bar{R}_{K}-R_{K}\right\|_{K}\right) .
$$


Thanks to the triangle inequality, one finds

$$
\begin{aligned}
\left\|H_{K} R_{K}\right\|_{K} & \leq\left\|H_{K} \bar{R}_{K}\right\|_{K}+\left\|H_{K}\left(\bar{R}_{K}-R_{K}\right)\right\|_{K} \\
& \leq C\left(\left\|\nabla\left(\mathbf{u}-\mathbf{u}_{H}^{v}\right)\right\|_{K}+\left\|p-p_{H}^{v}\right\|_{K}+\| H_{K}\left(\bar{R}_{K}-R_{K} \|_{K}\right)\right) .
\end{aligned}
$$

That is, the proof of (3.7) is completed.

Similar to the proof of (3.7), using (1.2), Lemmas 2.1 and 3.3 and noting [23, 37], we obtain

$$
\begin{aligned}
& \left|\int_{E} v \nabla\left(\mathbf{u}-\mathbf{u}_{H}^{v}\right) \cdot \nabla\left(b_{E} \bar{J}_{E}\right) d x-\int_{E}\left(p-p_{H}^{v}\right) \cdot \nabla \cdot\left(b_{E} \bar{J}_{E}\right) d x\right| \\
& \quad \leq C H_{E}^{-1 / 2}\left(\left\|\nabla\left(\mathbf{u}-\mathbf{u}_{H}^{v}\right)\right\|_{\omega_{E}}+\left\|p-p_{H}^{v}\right\|_{\omega_{E}}\right)\left\|\bar{J}_{E}\right\|_{E}, \\
& \left|\int_{E} b_{E} \bar{J}_{E}\left(\bar{J}_{E}-J_{E}\right) d s\right| \leq C\left\|\bar{J}_{E}-J_{E}\right\|_{E}\left\|b_{E} \bar{J}_{E}\right\|_{E}=0, \\
& \left|\int_{E}\left(f+v \Delta \mathbf{u}_{H}^{v}-\mathbf{a} \cdot \nabla \mathbf{u}_{H}^{v}-\nabla p_{H}^{v}\right) \cdot b_{E} \bar{J}_{E} d x+\int_{E}\left(\mathbf{a} \cdot \nabla\left(\mathbf{u}-\mathbf{u}_{H}^{v}\right)\right) \cdot b_{E} \bar{J}_{E} d x\right| \\
& \quad \leq C\left(H_{E}^{1 / 2}\left\|R_{K}\right\|_{\omega_{E}}+H_{E}^{1 / 2} C_{D}|\mathbf{a}|_{\infty}\left\|\nabla\left(\mathbf{u}-\mathbf{u}_{H}^{v}\right)\right\|_{\omega_{E}}\right)\left\|\bar{J}_{E}\right\|_{E} .
\end{aligned}
$$

Thus we have

$$
\begin{aligned}
\left\|\bar{J}_{E}\right\|_{E}^{2}= & \frac{3}{2}\left(\int_{E} J_{E} b_{E} \bar{J}_{E} d s+\int_{E} b_{E} \bar{J}_{E}\left(\bar{J}_{E}-J_{E}\right) d s\right) \\
\approx & \int_{E}\left(-v \Delta \mathbf{u}_{H}^{v}+\nabla p_{H}^{v}\right) \cdot b_{E} \bar{J}_{E} d x-\int_{E}\left(v \nabla \mathbf{u}_{H}^{v}-p_{H}^{v}\right) \cdot \nabla\left(b_{E} \bar{J}_{E}\right) d x+\int_{E} b_{E} \bar{J}_{E}\left(\bar{J}_{E}-J_{E}\right) d s \\
\leq & \mid \int_{E} v \nabla\left(\mathbf{u}-\mathbf{u}_{H}^{v}\right) \cdot \nabla\left(b_{E} \bar{J}_{E}\right) d x-\int_{E}\left(p-p_{H}^{v}\right) \cdot \nabla \cdot\left(b_{E} \bar{J}_{E}\right) d x+\int_{E} b_{E} \bar{J}_{E}\left(\bar{J}_{E}-J_{E}\right) d s \\
& -\int_{E}\left(f+v \Delta \mathbf{u}_{H}^{v}-\mathbf{a} \cdot \nabla \mathbf{u}_{H}^{v}-\nabla p_{H}^{v}\right) \cdot b_{E} \bar{J}_{E} d x+\int_{E}\left(\mathbf{a} \cdot \nabla\left(\mathbf{u}-\mathbf{u}_{H}^{v}\right)\right) \cdot b_{E} \bar{J}_{E} d x \mid \\
\leq & C\left[H_{E}^{-1 / 2}\left(\left\|\nabla\left(\mathbf{u}-\mathbf{u}_{H}^{v}\right)\right\|_{\omega_{E}}+\left\|p-p_{H}^{v}\right\|_{\omega_{E}}\right)+H_{E}^{1 / 2}\left\|R_{K}\right\|_{\omega_{E}}\right]\left\|\bar{J}_{E}\right\|_{E} \cdot
\end{aligned}
$$

Then (3.8) is obtained by the triangle inequality, (3.7), and (3.9).

Using Lemma 3.4 and taking a sum over all elements and edges, we obtain the following result.

Theorem 3.5 Let $(\mathbf{u}, p) \in X \times M$ and $\left(\mathbf{u}_{H}^{v}, p_{H}^{v}\right) \in X_{H} \times M_{H}$ be the solutions of problems (1.2) and (2.10), respectively, then we have

$$
\eta_{H} \leq C\left(\left\|\nabla\left(\mathbf{u}-\mathbf{u}_{H}^{v}\right)\right\|_{0}+\left\|p-p_{H}^{v}\right\|_{0}+O S C_{H}\right)
$$

\section{Discrete local lower bound}

In this section, we consider the discrete local lower bound for $\left\|\nabla\left(\mathbf{u}_{h}^{v}-\mathbf{u}_{H}^{v}\right)\right\|_{0}+\left\|p_{h}^{v}-p_{H}^{v}\right\|_{0}$ between two conforming triangulations $\mathcal{T}_{h}$ and $\mathcal{T}_{H}$ and the corresponding finite element spaces $X_{H} \times M_{H} \subset X_{h} \times M_{h}$. Furthermore, the interior node property holds on each edge 


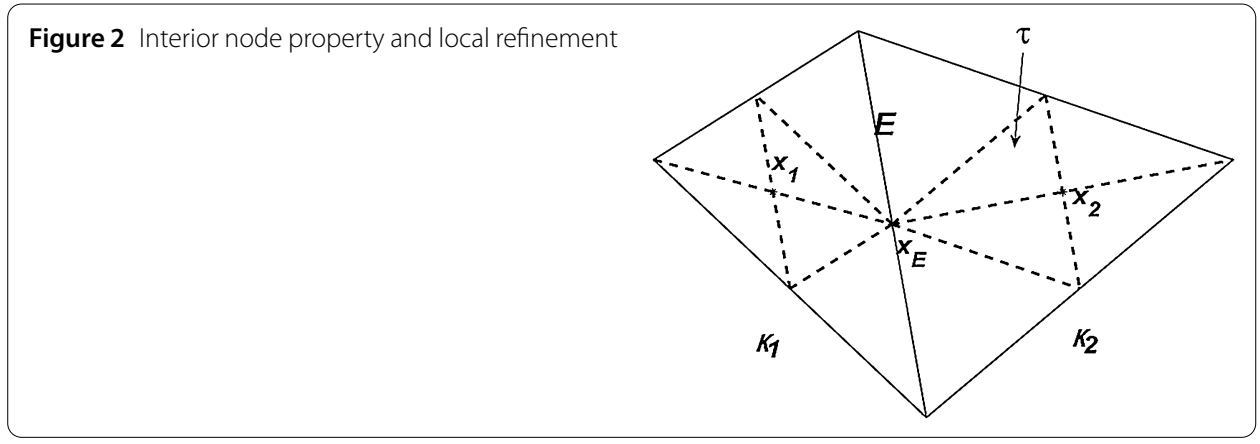

$E$ of $\mathcal{T}_{H}$ (see [37]): The interior of $E=\partial K_{1} \cap \partial K_{2}\left(K_{1}, K_{2} \in \mathcal{T}_{H}\right)$ contains at least one vertex of $\mathcal{T}_{H}$, see Fig. 2.

Compared with the results of the residual-based error estimator in the previous section, we focus on the discrete local lower bound on the domain $\omega_{E}$ for each $E \in \mathcal{E}_{H}$.

Lemma 4.1 Under the assumption of the interior node property, let $\left(\mathbf{u}_{h}^{v}, p_{h}^{v}\right) \in X_{h} \times M_{h}$ and $\left(\mathbf{u}_{H}^{v}, p_{H}^{v}\right) \in X_{H} \times M_{H}$ be the stabilized finite volume solutions of problem (2.10) on $\mathcal{T}_{h}$ and $\mathcal{T}_{H}$, respectively. Then, for $K_{i} \in \omega_{E}(i=1,2)$, we have

$$
\left\|H_{K_{i}} R_{K_{i}}\right\|_{0, K_{i}} \leq C\left(\left\|\nabla\left(\mathbf{u}_{h}^{v}-\mathbf{u}_{H}^{v}\right)\right\|_{0, K_{i}}+\left\|p_{h}^{v}-p_{H}^{v}\right\|_{0, K_{i}}+O S C_{H}(K)\right) .
$$

Proof The triangle inequality gives

$$
\left\|R_{K_{i}}\right\|_{0, K_{i}} \leq\left\|R_{K_{i}}-\bar{R}_{K_{i}}\right\|_{0, K_{i}}+\left\|\bar{R}_{K_{i}}\right\|_{0, K_{i}}
$$

For the second term on the right-hand side of (4.1), choosing $\mathbf{v}_{i}=I_{H} \mathbf{b}_{\mathbf{K}_{\mathbf{i}}}, \mathbf{v}_{i}^{*}=\Gamma_{H} \mathbf{v}_{i} \in X_{H}^{*}$ and applying Lemmas 2.4 and 3.3, the inverse inequality, and the interior node property, we see that

$$
\begin{aligned}
\left\|\bar{R}_{K_{i}}\right\|_{0, K_{i}}^{2} \sim & \left(\bar{R}_{K_{i}}, \mathbf{v}_{i}^{*} \bar{R}_{K_{i}}\right) \\
= & \left(f+v \Delta \mathbf{u}_{H}^{v}-\mathbf{a} \cdot \nabla \mathbf{u}_{H}^{v}-\nabla p_{H}^{v}, \mathbf{v}_{i}^{*} \bar{R}_{K_{i}}\right)-\left(R_{K_{i}}-\bar{R}_{K_{i}}, \mathbf{v}_{i}^{*} \bar{R}_{K_{i}}\right) \\
= & A\left(\mathbf{u}_{h}^{v}-\mathbf{u}_{H}^{v}, \mathbf{v}_{i}^{*} \bar{R}_{K_{i}}\right)+D\left(p_{h}^{v}-p_{H}^{v}, \mathbf{v}_{i}^{*} \bar{R}_{K_{i}}\right)+\left(\mathbf{a} \cdot \nabla\left(\mathbf{u}_{h}^{v}-\mathbf{u}_{H}^{v}\right), \mathbf{v}_{i}^{*} \bar{R}_{K_{i}}\right) \\
& -\left(R_{K_{i}}-\bar{R}_{K_{i}}, \mathbf{v}_{i}^{*} \bar{R}_{K_{i}}\right) \\
= & v\left(\nabla\left(\mathbf{u}_{h}^{v}-\mathbf{u}_{H}^{v}\right), \nabla\left(I_{H} \mathbf{b}_{K_{\mathbf{i}}} \bar{R}_{K_{i}}\right)\right)+\left(\nabla \cdot\left(I_{H} \mathbf{b}_{K_{i}} \bar{R}_{K_{i}}\right), p_{h}^{v}-p_{H}^{v}\right) \\
& +\left(\mathbf{a} \cdot \nabla\left(\mathbf{u}_{h}^{v}-\mathbf{u}_{H}^{v}\right), \mathbf{v}_{i}^{*} \bar{R}_{K_{i}}\right)-\left(R_{K_{i}}-\bar{R}_{K_{i}}, \mathbf{v}_{i}^{*} \bar{R}_{K_{i}}\right) \\
\leq & C\left(H_{K_{i}}^{-1}\left(\left\|\nabla\left(\mathbf{u}_{h}^{v}-\mathbf{u}_{H}^{v}\right)\right\|_{0, K_{i}}+\left\|p_{h}^{v}-p_{H}^{v}\right\|_{0, K_{i}}\right)\right. \\
& \left.+C_{D}|\mathbf{a}|_{\infty}\left\|\nabla\left(\mathbf{u}_{h}^{v}-\mathbf{u}_{H}^{v}\right)\right\|_{0, K_{i}}+\left\|R_{K_{i}}-\bar{R}_{K_{i}}\right\|_{0, K_{i}}\right)\left\|\bar{R}_{K_{i}}\right\|_{0, K_{i}} .
\end{aligned}
$$

Therefore, thanks to (4.1) we have

$$
\left\|R_{K_{i}}\right\|_{0, K_{i}} \leq C\left(H_{K_{i}}^{-1}\left(\left\|\nabla\left(\mathbf{u}_{h}^{v}-\mathbf{u}_{H}^{v}\right)\right\|_{0, K_{i}}+\left\|p_{h}^{v}-p_{H}^{v}\right\|_{0, K_{i}}\right)+\left\|R_{K_{i}}-\bar{R}_{K_{i}}\right\|_{0, K_{i}}\right) .
$$

Multiplying the above inequality by $H_{K_{i}}$, a straightforward computation gives the desired result. 
Lemma 4.2 Under the assumption of the interior node property, let $\left(\mathbf{u}_{h}^{v}, p_{h}^{v}\right) \in X_{h} \times M_{h}$ and $\left(\mathbf{u}_{H}^{v}, p_{H}^{v}\right) \in X_{H} \times M_{H}$ be the stabilized finite volume solutions of problem (2.10) on $\mathcal{T}_{h}$ and $\mathcal{T}_{H}$, respectively. Then, for $E \in \mathcal{E}_{H}$, we have

$$
\left\|H_{E}^{1 / 2} J_{E}\right\|_{0, E} \leq C\left(\left\|\nabla\left(\mathbf{u}_{h}^{v}-\mathbf{u}_{H}^{v}\right)\right\|_{0, K_{i}}+\left\|p_{h}^{v}-p_{H}^{v}\right\|_{0, K_{i}}+O S C_{H}(K)\right) .
$$

Proof Setting $\mathbf{v}_{E}=I_{H} b_{E}$ and $\mathbf{v}_{E}^{*}=\Gamma_{H} \mathbf{v}_{E}$ and noting that $\left[p_{H}^{v} I \cdot \mathbf{n}\right]_{E}=0$, we have

$$
\left\|J_{E}\right\|_{0, E}^{2} \sim\left(J_{E}, \mathbf{v}_{E}^{*} J_{E}\right)=\left(v \frac{\partial \mathbf{u}_{H}^{v}}{\partial \mathbf{n}}-p_{H}^{v} I \cdot \mathbf{n}, \mathbf{v}_{E}^{*} J_{E}\right)
$$

Using the Green's formula and the divergence theorem on each $Q \in K \cap \bar{K}$, we get (see Fig. 1)

$$
\begin{aligned}
& \left(v \frac{\partial \mathbf{u}_{H}^{v}}{\partial \mathbf{n}}, \mathbf{v}_{E}^{*}\right)_{E}=-\int_{\partial \widetilde{K}_{i}} v \frac{\partial \mathbf{u}_{H}^{v}}{\partial \mathbf{n}} \cdot \mathbf{v}_{E}^{*} d s+\int_{Q} v \Delta \mathbf{u}_{H}^{v} \cdot \mathbf{v}_{E}^{*} d x, \\
& \left(p_{H}^{v} I \cdot \mathbf{n}, \mathbf{v}_{E}^{*}\right)_{E}=-\int_{\partial \widetilde{K}_{i}} p_{H}^{v} \cdot \mathbf{n v}_{E}^{*} d s+\int_{Q} \nabla p_{H}^{v} \cdot \mathbf{v}_{E}^{*} d x .
\end{aligned}
$$

Using the above equalities and recalling the definitions of $A(\cdot, \cdot)$ and $D(\cdot, \cdot)$, we see that

$$
\begin{aligned}
\left(v \frac{\partial \mathbf{u}_{H}^{v}}{\partial \mathbf{n}}-p_{H}^{v} I \cdot \mathbf{n}, \mathbf{v}_{E}^{*}\right)_{E} & =-\int_{\partial \widetilde{K}_{i}}\left(v \frac{\partial \mathbf{u}_{H}^{v}}{\partial \mathbf{n}}-p_{H}^{v} I \cdot \mathbf{n}\right) \cdot \mathbf{v}_{E}^{*} d s+\int_{Q}\left(v \Delta \mathbf{u}_{H}^{v}-\nabla p_{H}^{v}\right) \cdot \mathbf{v}_{E}^{*} d x \\
& =\int_{Q}\left(\mathbf{f}-\mathbf{a} \cdot \nabla \mathbf{u}_{H}^{v}+v \Delta \mathbf{u}_{H}^{v}-\nabla p_{H}^{v}\right) \cdot \mathbf{v}_{E}^{*} d x
\end{aligned}
$$

Due to supp $b_{E} \subset \omega_{E}$, multiplying $J_{E}$ to both sides of the above equality, we obtain

$$
\begin{aligned}
& \left|\int_{Q}\left(\mathbf{f}-\mathbf{a} \cdot \nabla \mathbf{u}_{H}^{v}+v \Delta \mathbf{u}_{H}^{v}-\nabla p_{H}^{v}\right) \cdot J_{E} \mathbf{v}_{E}^{*} d x\right| \\
& \quad \leq C\left\|R_{K}\right\|_{0, \omega_{E}}\left\|J_{E} \mathbf{v}_{E}^{*}\right\|_{0, \omega_{E}} \leq C H_{E}^{1 / 2}\left\|R_{K}\right\|_{0, \omega_{E}}\left\|J_{E}\right\|_{0, \omega_{E}} .
\end{aligned}
$$

Combine (4.2) with (4.3) to arrive at

$$
\left\|H_{E}^{1 / 2} J_{E}\right\|_{0, E} \leq C\left\|H_{K_{i}} R_{K}\right\|_{0, \omega_{E}} .
$$

The proof is completed by using Lemma 4.1 and (4.4).

Finally, from Lemmas 4.1 and 4.2, we obtain the main result of this section.

Theorem 4.3 Under the assumption of the interior node property, let $\left(\mathbf{u}_{h}^{v}, p_{h}^{v}\right) \in X_{h} \times M_{h}$ and $\left(\mathbf{u}_{H}^{v}, p_{H}^{v}\right) \in X_{H} \times M_{H}$ be the stabilized finite volume solutions of problem (2.10) on $\mathcal{T}_{h}$ and $\mathcal{T}_{H}$, respectively. Then we have

$$
\eta_{H} \leq C_{6}\left(\left\|\nabla\left(\mathbf{u}_{h}^{v}-\mathbf{u}_{H}^{v}\right)\right\|_{0}+\left\|p_{h}^{v}-p_{H}^{v}\right\|_{0}+O S C_{H}\right) .
$$




\section{Adaptive finite volume method and convergence analysis}

In this section, we develop an adaptive finite volume method based on the local error estimator presented in the previous sections. The techniques are adopted from [23, 37] for the second order elliptic problem. For the Oseen equations, the adaptive finite volume method can be divided into several steps. For the sake of convenience, we set $\mathcal{T}_{k}$, $k=0,1,2, \ldots$, to be a sequence of shape regular triangulations and $\left(\mathbf{u}_{k}^{v}, p_{k}^{v}\right), k=0,1,2, \ldots$, to be a sequence of stabilized finite volume solutions on the corresponding nested finite element spaces generated by the adaptive finite volume method. We have developed the quasi-residual type of the a posteriori error estimator and established the upper and lower bounds between the exact solution and the finite volume solutions, and the discrete local lower bounds between the approximate solutions on meshes $\mathcal{T}_{H}$ and $\mathcal{T}_{h}$ in Sects. 3 and 4.

\subsection{Adaptive finite volume method}

First, we choose parameters $\theta_{1}, \theta_{2} \in(0,1)$ and an initial mesh $\mathcal{T}_{0}$ with mesh size $h_{0}=H$.

Step 1 . Solve and estimate.

1. Solve the following stabilized finite volume formulation to find $\left(\mathbf{u}_{k}^{v}, p_{k}^{v}\right)$ :

$$
\mathcal{C}\left(\left(\mathbf{u}_{k}^{v}, p_{k}^{v}\right),\left(\mathbf{v}_{k}, q_{k}\right)\right)=\left(\mathbf{f}, v_{k}^{*}\right)
$$

2. Compute the residual error estimator $\eta_{h_{k}}$.

Step 2. Local refinement.

1. Determine a suitable adaptive refinement for an update. Let $\mathcal{U}_{k}$ be the minimal edge set required refinement by satisfying the following marking strategy:

$$
\theta_{1} \eta_{h_{k}} \leq \eta_{h_{k}}\left(\omega_{\mathcal{U}_{k}}\right), \quad \theta_{2} \operatorname{OSC}_{h_{k}} \leq \operatorname{OSC}_{h_{k}}\left(\omega_{\mathcal{U}_{k}}\right)
$$

2. Refinement and completion. Let $\mathcal{T}_{k+1}$ be the refinement of $\mathcal{T}_{k}$. Refine each element $K \in \omega_{\mathcal{U}_{k}}$ and complete the hanging points, such that $\mathcal{T}_{k+1}$ is a conforming triangulation.

Step 3. Cycle criterion: For a sufficiently small tolerance $\varepsilon>0$, if $\eta_{h_{k}} \leq \varepsilon$, stop the computation. Otherwise, set $k=k+1$ and go to Step 2 .

The convergence analysis for the adaptive stabilized finite volume method will be discussed in what follows. It is known that the convergence analysis of finite element method requires the orthogonality property $[23,37]$. However, the corresponding property loses effectiveness for the finite volume method due to the test and trial functions that belong to different spaces for the present method.

Theorem 5.1 Set $\left(\mathbf{u}_{h}^{v}, p_{h}^{v}\right) \in X_{h} \times M_{h}$ and $\left(\mathbf{u}_{H}^{v}, p_{H}^{v}\right) \in X_{H} \times M_{H}$ be the stabilized finite volume solutions of problem (2.10) on $\mathcal{T}_{h}$ and $\mathcal{T}_{H}$, respectively. Denote $e_{h}=\mathbf{u}_{h}^{v}-\mathbf{u}_{H}^{v}$ and $\epsilon_{h}=p_{h}^{v}-p_{H}^{v}$. Then we have

$$
\begin{aligned}
& v\left(\nabla\left(\mathbf{u}-\mathbf{u}_{H}^{v}\right), \nabla e_{h}\right)-\left(\nabla \cdot e_{h}, p-p_{H}^{v}\right)+\left(\nabla \cdot\left(\mathbf{u}-\mathbf{u}_{H}^{v}\right), \epsilon_{h}\right)+\left(\mathbf{a} \cdot \nabla\left(\mathbf{u}-\mathbf{u}_{H}^{v}\right), e_{h}\right) \\
& \quad \leq C\left(\left\|\nabla\left(\mathbf{u}_{h}^{v}-\mathbf{u}_{H}^{v}\right)\right\|_{0}+\left\|p_{h}^{v}-p_{H}^{v}\right\|_{0}\right) \cdot O S C_{H} .
\end{aligned}
$$

Proof Choosing $(\mathbf{v}, q)=\left(\mathbf{v}_{H}, q_{H}\right)$ in (3.1), it follows from Lemma 2.3 and Theorem 3.1 that

$$
\left|\int_{K} R_{K} \cdot\left(\mathbf{v}_{H}-\mathbf{v}_{H}^{*}\right) d x\right|=\left|\int_{K}\left(R_{K}-\bar{R}_{K}\right) \cdot\left(\mathbf{v}_{H}-\mathbf{v}_{H}^{*}\right) d x\right| \leq C H_{K}\left\|R_{K}-\bar{R}_{K}\right\|_{0, K}\left\|\mathbf{v}_{H}\right\|_{1, K},
$$




$$
\begin{aligned}
& \left|\int_{K} \nabla \cdot \mathbf{u}_{H}^{v} q_{H} d x\right| \leq\left\|\nabla \cdot \mathbf{u}_{H}^{v}\right\|_{0, K}\left\|q_{H}\right\|_{0, K}, \quad \forall K \in \mathcal{T}_{H}, \\
& \left|\int_{E} J_{E}\left(\mathbf{v}-\mathbf{v}_{H}^{*}\right) d s\right|=\left|\int_{E}\left(J_{E}-\bar{J}_{E}\right)\left(\mathbf{v}-\mathbf{v}_{H}^{*}\right) d s\right|=0, \quad \forall E \in \partial K .
\end{aligned}
$$

Summing up over all $K \in \mathcal{T}_{H}$ and $E \in \mathcal{E}_{H}$ with $\left(\mathbf{v}_{H}, q_{H}\right)=\left(e_{h}, \epsilon_{h}\right)$ and using the definition of $O S C_{H}$, we obtain the desired result.

\subsection{Error reduction of two successive steps}

The convergence property guarantees that the iterative loop terminates in a finite number of steps starting from an initial coarse mesh. Now, we investigate the adaptive stabilized finite volume method for the Oseen equations in terms of an error reduction between two successive steps. The mathematical induction argument can be used to obtain the error reduction in a finite number of steps.

Lemma 5.2 Let $\left(\mathbf{u}_{h}^{v}, p_{h}^{v}\right) \in X_{h} \times M_{h}$ and $\left(\mathbf{u}_{H}^{v}, p_{H}^{v}\right) \in X_{H} \times M_{H}$ be the stabilized finite volume solutions of problem (2.10) on $\mathcal{T}_{h}$ and $\mathcal{T}_{H}$, respectively. Set $H=\max _{K \in \mathcal{T}_{H}} H_{K}$. Then there exists a constant $\rho_{1} \in(0,1)$ such that

$$
O S C_{h}^{2} \leq \rho_{1} O S C_{H}^{2}
$$

Proof Here, we only estimate the term $R_{\tau}, \tau \in \mathcal{T}_{h}$ (see Fig. 2). Others can be bounded with a similar argument. For the residual on $\tau \subset K \subset \mathcal{T}_{H}$, we have

$$
R_{\tau}=R_{\tau}^{H}-\nabla\left(\nabla p_{h}^{v}-\nabla p_{H}^{v}\right)-\mathbf{a} \cdot\left(\nabla \mathbf{u}_{h}^{v}-\nabla \mathbf{u}_{H}^{v}\right)
$$

where $R_{\tau}$ and $R_{\tau}^{H}$ are the error estimators on mesh $\mathcal{T}_{h}$ with solutions $\left(\mathbf{u}_{h}^{v}, p_{h}^{v}\right)$ and $\left(\mathbf{u}_{H}^{v}, p_{H}^{v}\right)$, respectively. Using the definition of $R_{\tau},(5.1)$ and the Young inequality, we see that

$$
O S C_{h}^{2}(K)=\sum_{\tau \subset K} h_{\tau}^{2}\left\|R_{\tau}-\bar{R}_{\tau}\right\|_{0, \tau}^{2}+\sum_{\tau \subset K}\left\|\nabla \cdot \mathbf{u}_{h}^{v}\right\|_{0, \tau}^{2}
$$

Since $\nabla \epsilon_{h}=\nabla\left(p_{h}^{v}-p_{H}^{v}\right)$ is a constant, thus $\nabla \epsilon_{h}-\overline{\nabla \epsilon_{h}}=0$. Setting $\gamma_{0} \in(0,1)$ such that $h_{\tau} \leq \gamma_{0} H_{K}$ and $K \in \omega_{\mathcal{U}_{H}}$, we define a refinement factor by

$$
\gamma_{K}= \begin{cases}\gamma_{0}, & \text { in } K \in \omega_{\mathcal{U}_{H}}, \\ 1, & \text { in } \Omega \backslash \omega_{\mathcal{U}_{H}} .\end{cases}
$$

Thanks to (2.4), (5.2), the making strategy on local refinement and straightforward computation, the above inequality restricted to the mesh $\mathcal{T}_{H}$ can be rewritten as

$$
\begin{aligned}
O S C_{h}^{2} & =\sum_{K \in \mathcal{T}_{H}} \sum_{\tau \subset K} \operatorname{OSC}_{h}^{2}(\tau) \\
& \leq \sum_{K \in \mathcal{T}_{H}} \gamma_{K}^{2} H_{K}^{2}\left\|R_{\tau}-\bar{R}_{\tau}\right\|_{0, K}^{2}+\sum_{K \in \mathcal{T}_{H}}\left\|\nabla \cdot \mathbf{u}_{h}^{v}\right\|_{0, K}^{2} \\
& \leq \sum_{K \in \mathcal{T}_{H} \backslash \omega \mathcal{U}_{K}} \gamma_{0}^{2}\left(H_{K}^{2}\left\|R_{\tau}-\bar{R}_{\tau}\right\|_{0, K}^{2}+\sum_{K \in \mathcal{T}_{H}}\left\|\nabla \cdot \mathbf{u}_{h}^{v}\right\|_{0, K}^{2}\right)
\end{aligned}
$$




$$
\begin{aligned}
& +\sum_{K \in \omega \mathcal{U}_{K}} \gamma_{0}^{2}\left(H_{K}^{2}\left\|R_{\tau}-\bar{R}_{\tau}\right\|_{0, K}^{2}+\sum_{K \in \mathcal{T}_{H}}\left\|\nabla \cdot \mathbf{u}_{h}^{v}\right\|_{0, K}^{2}\right)+\left(1-\gamma_{0}^{2}\right) \sum_{K \in \mathcal{T}_{H}}\left\|\nabla \cdot \mathbf{u}_{h}^{v}\right\|_{0, K}^{2} \\
\leq & 2 O S C_{H}^{2}-\left(1-\gamma_{0}^{2}\right) \sum_{K \in \mathcal{U}_{K}} O S C_{H}^{2}(\tau) \\
\leq & \left(2-\left(1-\gamma_{0}^{2}\right) \theta_{2}^{2}\right) O S C_{H}^{2} .
\end{aligned}
$$

Thus, the desired result is obtained by choosing $\rho_{1}=2-\left(1-\gamma_{0}^{2}\right) \theta_{2}^{2} \in(0,1)$ in the above inequality.

Lemma 5.3 Let $\left(\mathbf{u}_{h}^{v}, p_{h}^{v}\right) \in X_{h} \times M_{h}$ and $\left(\mathbf{u}_{H}^{v}, p_{H}^{v}\right) \in X_{H} \times M_{H}$ be the stabilized finite volume solutions of problem (2.10) on $\mathcal{T}_{h}$ and $\mathcal{T}_{H}$, respectively. There exist constants $\gamma>0$ and $\rho_{0} \in(0,1)$ such that

$$
\left\|\mathbf{u}-\mathbf{u}_{h}^{v}\right\|_{1}+\left\|p-p_{h}^{v}\right\|_{0}+\gamma O S C_{h}^{2} \leq \rho_{0}\left(\left\|\mathbf{u}-\mathbf{u}_{H}^{v}\right\|_{1}+\left\|p-p_{H}^{v}\right\|_{0}+\gamma O S C_{H}^{2}\right) .
$$

Proof Using (1.3) and the Young inequality, we have

$$
\begin{aligned}
\| \mathbf{u}- & \mathbf{u}_{h}^{v}\left\|_{1}^{2}+\right\| p-p_{h}^{v} \|_{0}^{2} \\
= & \left\|\mathbf{u}-\mathbf{u}_{H}^{v}\right\|_{1}^{2}-\left\|\mathbf{u}_{h}^{v}-\mathbf{u}_{H}^{v}\right\|_{1}^{2}-2\left(\nabla\left(\mathbf{u}-\mathbf{u}_{h}^{v}\right), \nabla\left(\mathbf{u}_{h}^{v}-\mathbf{u}_{H}^{v}\right)\right) \\
& +\left\|p-p_{H}^{v}\right\|_{0}^{2}-\left\|p_{h}^{v}-p_{H}^{v}\right\|_{0}^{2}-2\left(p-p_{h}^{v}, p_{h}^{v}-p_{H}^{v}\right) \\
\leq & \left\|\mathbf{u}-\mathbf{u}_{H}^{v}\right\|_{1}^{2}+\left\|p-p_{H}^{v}\right\|_{0}^{2}-\left(\left\|\mathbf{u}_{h}^{v}-\mathbf{u}_{H}^{v}\right\|_{1}^{2}+\left\|p_{h}^{v}-p_{H}^{v}\right\|_{0}^{2}\right) \\
& +2\left(\left\|\mathbf{u}-\mathbf{u}_{h}^{v}\right\|_{1}+\left\|p-p_{h}^{v}\right\|_{0}\right) \cdot\left(\left\|\mathbf{u}_{h}^{v}-\mathbf{u}_{H}^{v}\right\|_{1}+\left\|p_{h}^{v}-p_{H}^{v}\right\|_{0}\right) \\
\leq & \left\|\mathbf{u}-\mathbf{u}_{H}^{v}\right\|_{1}^{2}+\left\|p-p_{H}^{v}\right\|_{0}^{2}-\left(\left\|\mathbf{u}_{h}^{v}-\mathbf{u}_{H}^{v}\right\|_{1}^{2}+\left\|p_{h}^{v}-p_{H}^{v}\right\|_{0}^{2}\right) \\
& +\beta^{-1} \mid v\left(\nabla\left(\mathbf{u}-\mathbf{u}_{h}^{v}\right), \nabla\left(\mathbf{u}_{h}^{v}-\mathbf{u}_{H}^{v}\right)\right)-\left(\nabla \cdot\left(\mathbf{u}_{h}^{v}-\mathbf{u}_{H}^{v}\right), p-p_{h}^{v}\right) \\
& +\left(\mathbf{a} \cdot \nabla\left(\mathbf{u}-\mathbf{u}_{h}^{v}\right), \mathbf{u}_{h}^{v}-\mathbf{u}_{H}^{v}\right)+\left(\nabla \cdot\left(\mathbf{u}-\mathbf{u}_{h}^{v}\right), p_{h}^{v}-p_{H}^{v}\right) \mid .
\end{aligned}
$$

By Theorem 5.1 and the Young inequality, we obtain

$$
\begin{aligned}
\beta^{-1} \mid & v\left(\nabla\left(\mathbf{u}-\mathbf{u}_{h}^{v}\right), \nabla\left(\mathbf{u}_{h}^{v}-\mathbf{u}_{H}^{v}\right)\right)-\left(\nabla \cdot\left(\mathbf{u}_{h}^{v}-\mathbf{u}_{H}^{v}\right), p-p_{h}^{v}\right) \\
& +\left(a \cdot \nabla\left(\mathbf{u}-\mathbf{u}_{h}^{v}\right), \mathbf{u}_{h}^{v}-\mathbf{u}_{H}^{v}\right)+\left(\nabla \cdot\left(\mathbf{u}-\mathbf{u}_{h}^{v}\right), p_{h}^{v}-p_{H}^{v}\right) \mid \\
\leq & C_{7}\left(\left\|\mathbf{u}_{h}^{v}-\mathbf{u}_{H}^{v}\right\|_{1}+\left\|p_{h}^{v}-p_{H}^{v}\right\|_{0}\right) \cdot O S C_{h} \\
\leq & \frac{1}{2}\left(\left\|\mathbf{u}_{h}^{v}-\mathbf{u}_{H}^{v}\right\|_{1}^{2}+\left\|p_{h}^{v}-p_{H}^{v}\right\|_{0}^{2}\right)+C_{7}^{2} O S C_{h}^{2} .
\end{aligned}
$$

Combine (5.3) with (5.4) and apply Lemma 5.2 to arrive at

$$
\begin{aligned}
& \left\|\mathbf{u}-\mathbf{u}_{h}^{v}\right\|_{1}^{2}+\left\|p-p_{h}^{v}\right\|_{0}^{2}+\gamma O S C_{h}^{2} \\
& \quad \leq\left\|\mathbf{u}-\mathbf{u}_{H}^{v}\right\|_{1}^{2}+\left\|p-p_{H}^{v}\right\|_{0}^{2}-\frac{1}{2}\left(\left\|\mathbf{u}_{h}^{v}-\mathbf{u}_{H}^{v}\right\|_{1}^{2}+\left\|p_{h}^{v}-p_{H}^{v}\right\|_{0}^{2}\right)+C_{8} O S C_{H}^{2},
\end{aligned}
$$


where $C_{8}=\left(\rho_{1} C_{7}^{2}+\gamma \rho_{1}\right)$. By the results of a lower bound of the solution on the initial mesh and Theorems 3.2 and 4.3, and the marking strategy, we have

$$
\begin{gathered}
\left\|\mathbf{u}-\mathbf{u}_{H}^{v}\right\|_{1}^{2}+\left\|p-p_{H}^{v}\right\|_{0}^{2} \leq C_{5}^{2} \eta_{H}^{2} \leq C_{5}^{2} \theta_{1}^{-2} \eta_{H}^{2}\left(\omega_{\mathcal{U}_{k}}\right) \\
\leq C_{9}\left(\left\|\mathbf{u}_{h}^{v}-\mathbf{u}_{H}^{v}\right\|_{1}^{2}+\left\|p_{h}^{v}-p_{H}^{v}\right\|_{0}^{2}+O S C_{H}^{2}\right),
\end{gathered}
$$

where $C_{9}=2 C_{5}^{2} C_{6}^{2} \theta_{1}^{-2}$. Substituting (5.6) into (5.5), we see that

$$
\begin{aligned}
\| \mathbf{u} & -\mathbf{u}_{h}^{v}\left\|_{1}^{2}+\right\| p-p_{h}^{v} \|_{0}^{2}+\gamma O S C_{h}^{2} \\
& \leq\left(1-\frac{1}{2 C_{9}}\right)\left(\left\|\mathbf{u}-\mathbf{u}_{H}^{v}\right\|_{1}^{2}+\left\|p-p_{H}^{v}\right\|_{0}^{2}\right)+\left(C_{8}+\frac{1}{2}\right) O S C_{H}^{2} .
\end{aligned}
$$

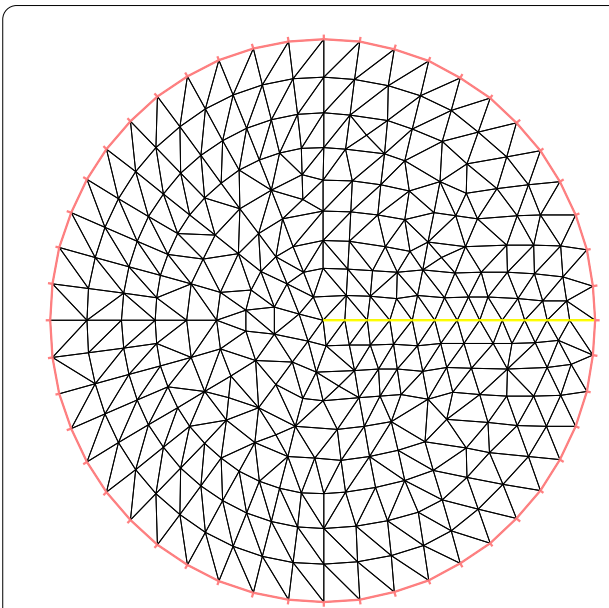

(a)

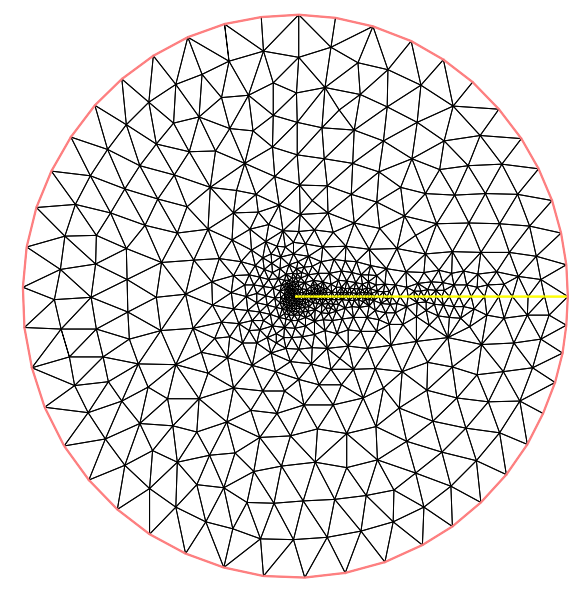

(c)

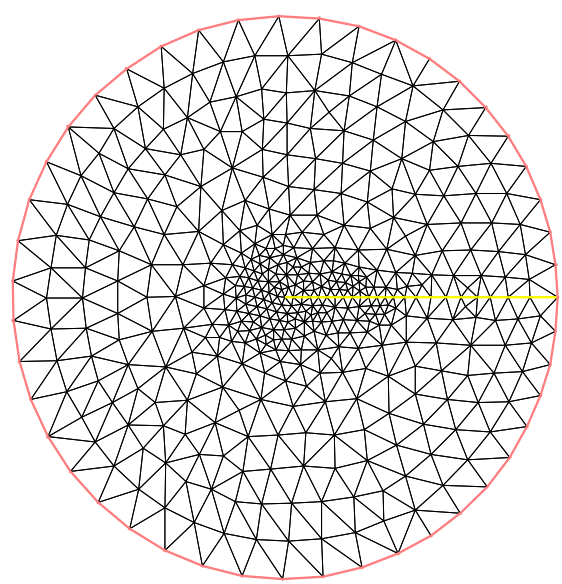

(b)

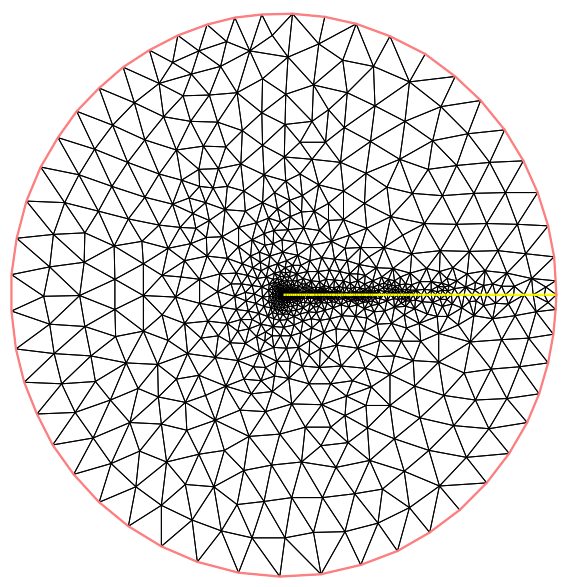

(d)

Figure 3 The mesh in adaptive computation. (a) Initial mesh. (b) Mesh after step 1. (c) Mesh after step 2. (d) Mesh after step 3 
Choose appropriate parameters $\theta_{1}>0$ and $\gamma>0$ such that

$$
1-\frac{1}{2 C_{9}}=1-\frac{\theta_{1}^{2}}{2 C_{5}^{2} C_{6}^{2}} \in(0,1) \quad \text { and } \quad C_{8}+\frac{1}{2}=\rho_{1} C_{7}^{2}+\gamma \rho_{1}+\frac{1}{2} \in(0,1) .
$$

Setting $\rho_{0}=\max \left\{1-\frac{1}{2 C_{9}}, C_{8}+\frac{1}{2}\right\}$, we complete the proof.

Using induction for a series of partitions $\mathcal{T}_{k}, k=0,1,2, \ldots$, we obtain the convergence of adaptive stabilized finite volume method.

Theorem 5.4 (Convergence analysis) Let $\left(\mathbf{u}_{k}^{v}, p_{k}^{v}\right), k=0,1,2, \ldots$, be a sequence of the corresponding stabilized finite volume solutions of problem (2.10) on $\mathcal{T}_{k}$, set the mesh size $H$ of $\mathcal{T}_{0}$ be sufficiently small. Then we have

$$
\left\|\mathbf{u}-\mathbf{u}_{h}^{v}\right\|_{1}+\left\|p-p_{h}^{v}\right\|_{0} \leq\left(\left\|\mathbf{u}-\mathbf{u}_{H}^{v}\right\|_{1}+\left\|p-p_{H}^{v}\right\|_{0}+\gamma O S C_{H}^{2}\right)^{1 / 2} \rho_{0}^{k} .
$$

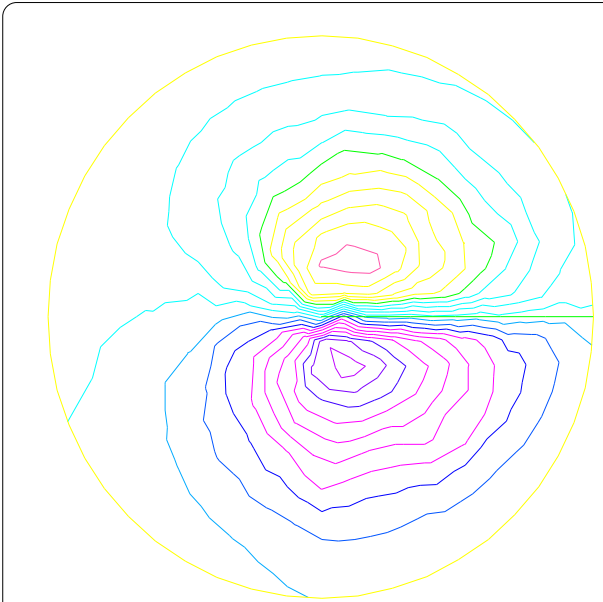

(a)

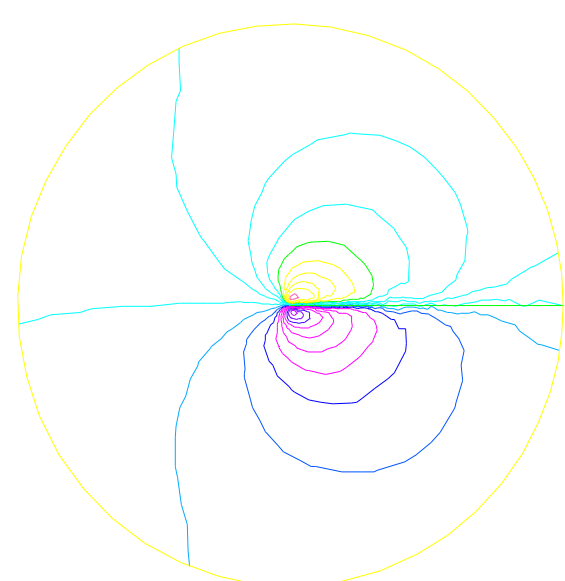

(c)

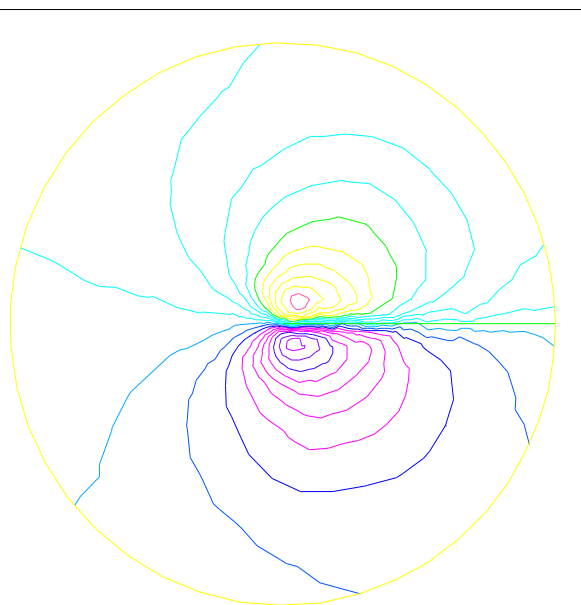

(b)

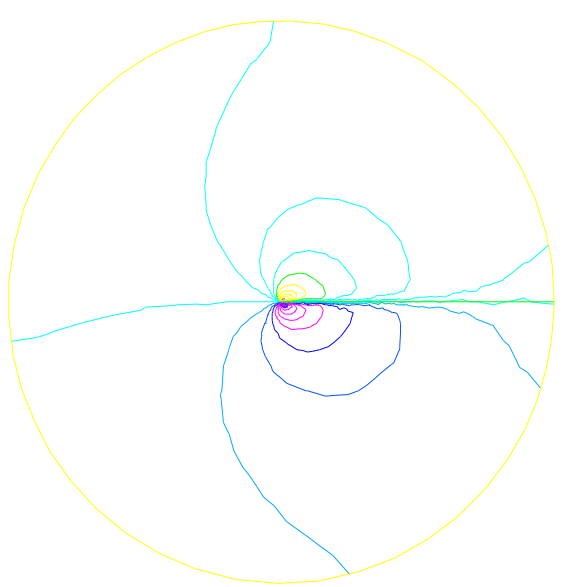

(d)

Figure 4 The contours of pressure. (a) Initial mesh. (b) Mesh after step 1. (c) Mesh after step 2. (d) Mesh after step 3 
From Theorem 5.4, we can see that our algorithm will terminate in a finite number of steps.

\section{Numerical validations}

In this section, we present two numerical examples to confirm our theoretical results and verify the efficiency of established a posteriori error estimators for the Oseen equations in the stabilized finite volume method. We solve the considered problem in the following adaptive strategy as presented in Sect. 5 .

(1) Give an initial triangulation $\mathcal{T}_{h}$ and a tolerance $\eta^{*}$. Solve the Oseen equations by the stabilized finite volume method on this partition.

(2) If $\left\{\sum_{K \in \mathcal{T}_{h}} \eta_{K}^{2}\right\}^{1 / 2} \leq \eta^{*}$, stop, we get the final numerical approximations. Otherwise, go to (3).

Table 1 Errors and the effective index on adaptive meshes with $v=1$

\begin{tabular}{rlllll}
\hline $\mathrm{N}$ & ratio & $\frac{\left\|u_{h}-u\right\|_{1}}{\|u\|_{1}}$ & $u_{H^{1}}$ rate & $\frac{\left\|p_{h}-p\right\|_{0}}{\|p\|_{0}}$ & $p_{L^{2}}$ rate \\
\hline 550 & 0.5437 & 0.372325 & & 0.875961 & \\
851 & 0.5404 & 0.323236 & 0.6478 & 0.737632 & 0.7875 \\
1234 & 0.5650 & 0.262181 & 1.1267 & 0.610745 & 1.0159 \\
1865 & 0.6187 & 0.217119 & 0.9133 & 0.501487 & 0.9545 \\
3011 & 0.6869 & 0.174988 & 0.9007 & 0.394036 & 1.0068 \\
\hline
\end{tabular}

Table 2 Error and the effective index on uniform meshes with $v=1$

\begin{tabular}{rlllll}
\hline $\mathrm{N}$ & ratio & $\frac{\left\|u_{h}-u\right\|_{1}}{\|u\|_{1}}$ & $u_{H^{1}}$ rate & $\frac{\left\|p_{h}-p\right\|_{0}}{\|p\|_{0}}$ & $p_{L^{2}}$ rate \\
\hline 550 & 0.5437 & 0.372325 & & 0.875961 & \\
857 & 0.5557 & 0.356411 & 0.1970 & 0.827581 & 0.2562 \\
1241 & 0.5491 & 0.340932 & 0.2399 & 0.796662 & 0.2057 \\
1872 & 0.5532 & 0.332744 & 0.1183 & 0.752626 & 0.2766 \\
3032 & 0.5721 & 0.309528 & 0.3001 & 0.703231 & 0.2815 \\
\hline
\end{tabular}

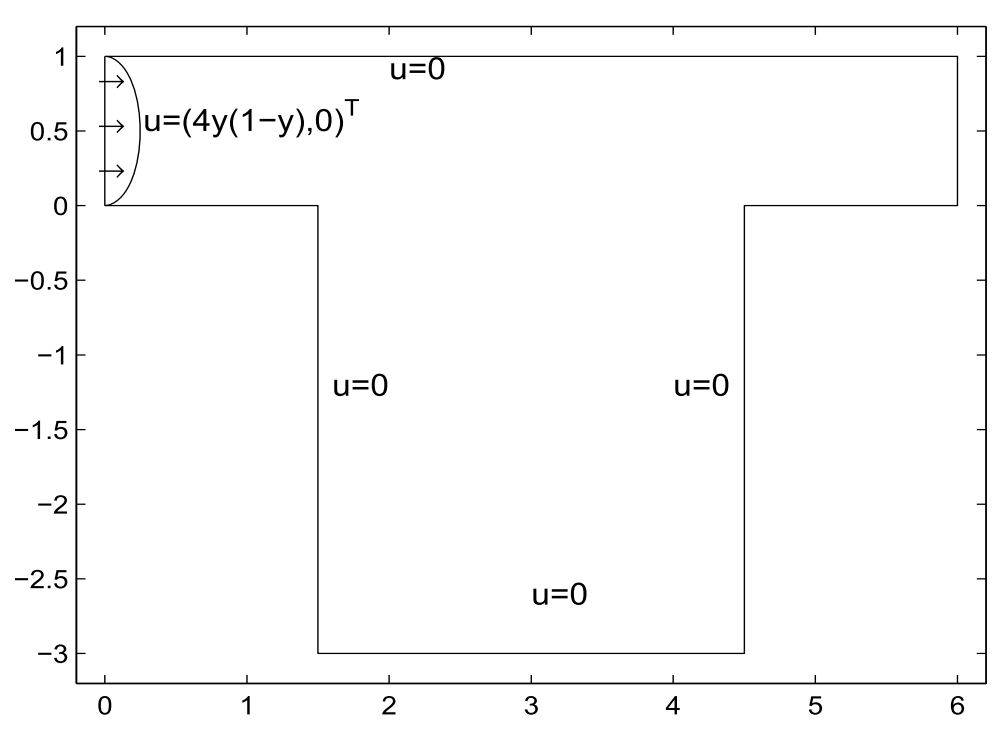

Figure 5 T-shape domain and the boundary conditions 
(3) Compute $\eta_{K}$ and $\left\{\sum_{K \in \mathcal{T}_{h}} \eta_{K}^{2}\right\}^{1 / 2}$, generate the new mesh size $h$, and re-compute $\left\{\sum_{K \in \mathcal{T}_{h}} \eta_{K}^{2}\right\}^{1 / 2}$ based on the new partition, then go to step (2).

\subsection{A singular problem with known solution}

In this test, we compute the Oseen equations in $\Omega$ with adaptive mesh and uniform mesh, where $\Omega$ is a disk of radius equal to 1 with a crack joining the center to the boundary as presented in [33], and the exact solutions for velocity and pressure are given by

$$
\begin{aligned}
& u_{1}=1.5 r^{\frac{1}{2}}(\cos (0.5 \theta)-\cos (1.5 \theta)), \\
& u_{2}=1.5 r^{\frac{1}{2}}(3 \sin (0.5 \theta)-\sin (1.5 \theta)), \\
& p=-6 r^{-\frac{1}{2}} \cos (0.5 \theta)
\end{aligned}
$$

where $(r, \theta)$ is a polar representation of a point in the disk and these solutions are singular at the end of the crack. $f$ is determined by (1.1), and non-homogeneous Dirichlet boundary conditions on the curved part of the boundary and homogeneous Dirichlet boundary conditions on the straight part of the boundary are given. We choose the advection coeffi-

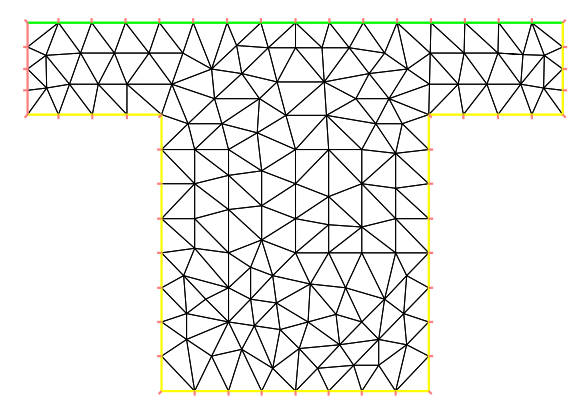

(a)

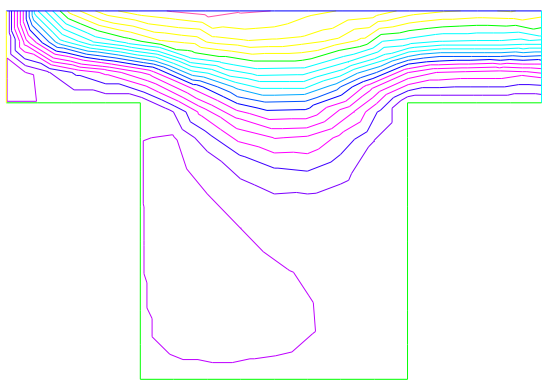

(c)

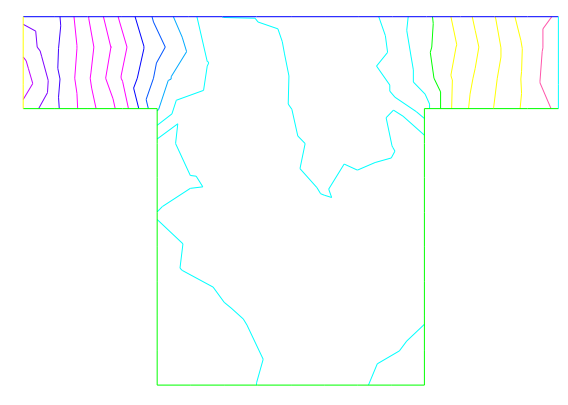

(b)

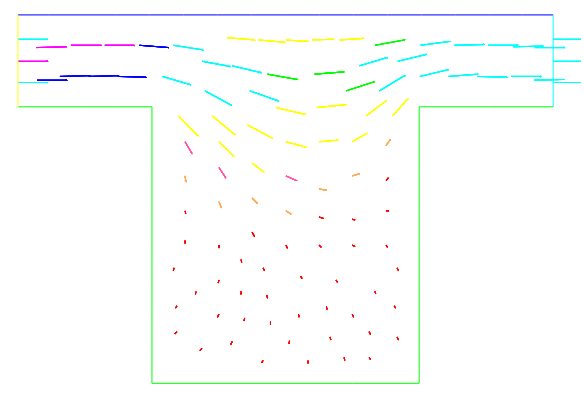

(d)

Figure 6 The mesh, the profiles of pressure and velocity, and the velocity vector with NT $=254$ 
cient $\mathbf{a}=\left(\begin{array}{l}1 \\ 1\end{array}\right)$ and $v=1$. We start our adaptive computation from the initial triangulations, as presented in Fig. 3(a) and refine three times shown in Fig. 3(b)-(d). It is observed that there are much more elements in the noncontinuous area than in the continuous area. Furthermore, the contours of the pressure near the crack become smooth as the number of triangles increases, see Fig. 4(a)-(d). In Tables 1-2, we provide the ratio between the error indicators and the discrete error, which is defined as the effective index in [19]. For a good estimator, this quantity should be a constant, independent of the mesh sizes. Here NT is the number of elements in the triangulations. The relative errors of velocity and pressure in different norms are presented in adaptive mesh and uniform mesh. The experimental convergence rates are given by $\alpha_{e_{\theta}}=\frac{2 * \log \left[\left\|e_{\theta}\left(\varepsilon_{1}\right)\right\|_{0} /\left\|e_{\theta}\left(\varepsilon_{2}\right)\right\| 0\right]}{\log \left[N T\left(\varepsilon_{1}\right) / N T\left(\varepsilon_{1}\right)\right]}$, where $\theta$ takes $u, p$.

Comparing Tables 1 and 2, we can see that the error estimator is good due to the ratio being a constant near 0.55 . Since the convergence order of the relative errors of velocity and pressure are $\mathcal{O}(h)$, then we can conclude that the convergence order of error estimator is $\mathcal{O}(h)$, which verifies the established theoretical analysis very well. On the other hand, the errors of adaptive procedures decrease much faster than those obtained by uniform ones. For example, the precision obtained with 3032 triangles in a

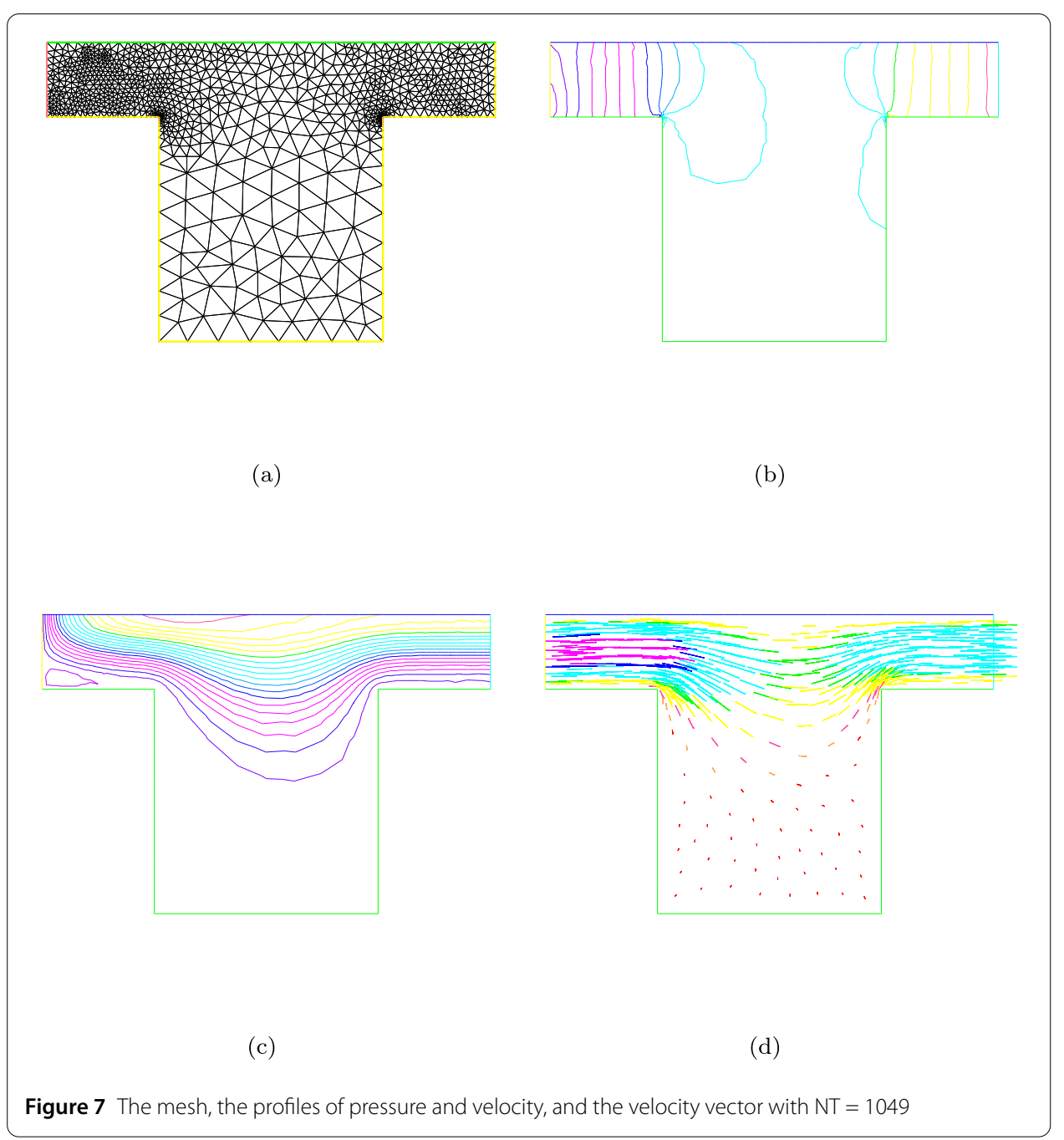


uniform mesh can be reached with 1234 triangles in an adaptive computation. This means we can save more work by the adaptive procedures than by the uniform procedures.

\subsection{T-shape domain model}

A T-shape domain model, as shown in Fig. 5, is a popular problem for testing the efficiency of the established a posteriori error estimators. In this test, we choose $u=(4 y(1-y), 0)$ on influx and natural boundary condition on outflux.

We start from the initial mesh with $h=0.25$, the corresponding profiles of velocity and pressure are presented in Fig. 6 . Then we note that the successive iterations of the adaptive strategies create more triangles in two corners of the T-shape domain as time increases, see Figs. 7-8. The profiles of velocity and pressure level lines are also presented with adaptive computations, see Figs. 7-8. As expected, the oscillations in the obtained pressure isovalues disappear and the velocity field becomes smooth. Finally, in order to show the prominent features of the established a posteriori error estimators, we compare the velocity and pressure contours obtained in the adaptive mesh with the uniform mesh using nearly the same number of triangles, see Figs. 8 and 9. From these figures, we

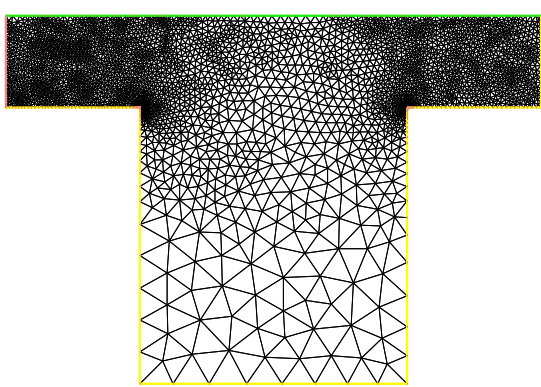

(a)

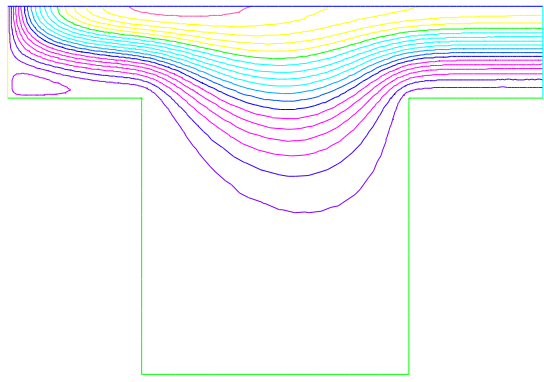

(c)

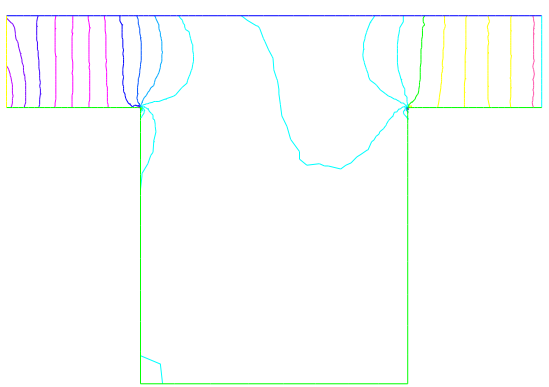

(b)

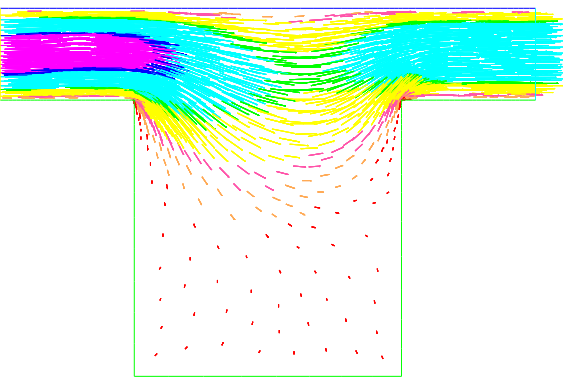

(d)

Figure 8 The mesh, the profiles of pressure and velocity, and the velocity vector with NT = 2821 


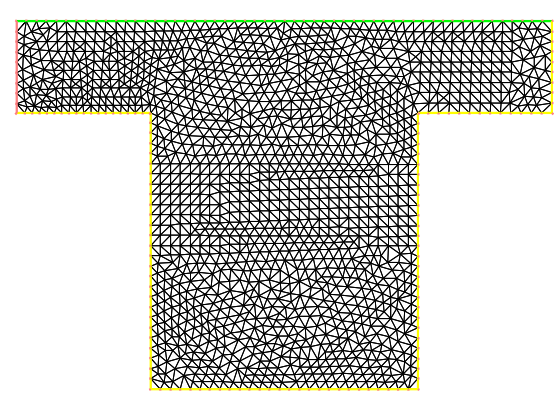

(a)

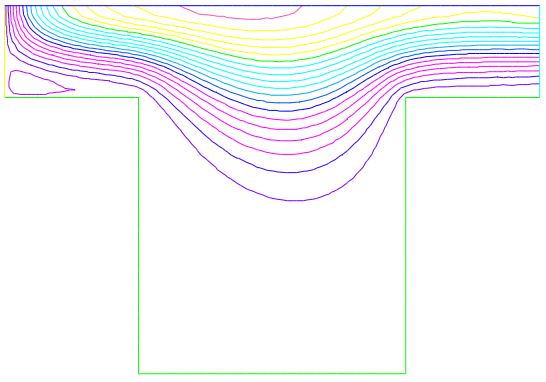

(c)

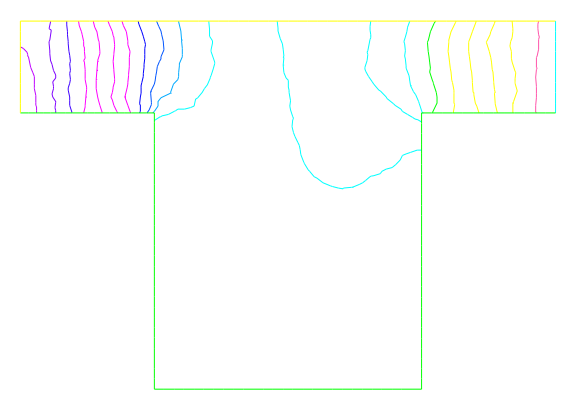

(b)

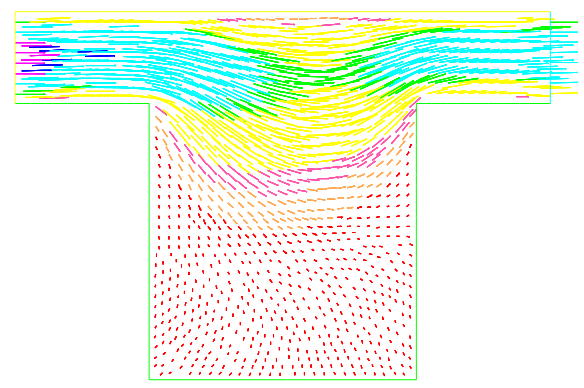

(d)

Figure 9 The mesh, the profiles of pressure and velocity, and the velocity vector with NT=2876

can see that the obtained results using the adaptive algorithm based on the a posteriori error estimators give more accurate approximation to the exact solutions in the critical regions.

\section{Conclusion}

In this paper, with the help of the pressure project method, we construct and analyze an adaptive stabilized finite volume method for the Oseen equations based on the lowest equal order finite element pair. A lower bound for the discrete local errors of numerical solutions is derived. Using the residual type a posteriori error estimator, the convergence of the adaptive stabilized finite volume algorithm is also established. Finally, some numerical results are presented to confirm the established theoretical findings and verify the efficiency of the developed error estimators. As an important model, the Oseen equations attract many researchers' attention in the computational field. How to extend the developed techniques of this paper to the Navier-Stokes equations will be discussed in our coming work. 
Acknowledgements

JXL and TZ express their gratitude to Shoujin Li for his discussions on this paper.

\section{Funding}

This work is supported by the National Natural Science Foundation of China (Nos. 11601410, 11601411), Shaanxi Natural Science Foundation (No. 2017JM1007), the Foundation for University Key Teacher by the Henan Province (2016GGJS-045), and the FDYS of Henan Polytechnic University (J2015-05).

\section{Abbreviations \\ Not applicable.}

Availability of data and materials

Not applicable.

\section{Ethics approval and consent to participate}

JXL gave some comments on the writing and revision of the paper, JXL also took part in the proof of error reduction. TZ wrote and revised the paper. They have no competing interests.

\section{Competing interests}

JXL and TZ declare that they have no competing interests.

\section{Consent for publication}

$J X L$ and TZ read and approved the final version of the manuscript.

\section{Authors' contributions}

JXL presented some comments on the writing and revising of the paper and took part in the proof of error reduction. TZ wrote and revised the paper. Both authors read and approved the final manuscript.

\section{Publisher's Note}

Springer Nature remains neutral with regard to jurisdictional claims in published maps and institutional affiliations.

\section{Received: 8 March 2018 Accepted: 31 July 2018 Published online: 22 August 2018}

\section{References}

1. Bi, C.J., Wang, C.: A posteriori error estimates of finite volume element method for second order quasilinear elliptic problems. Int. J. Numer. Anal. Model. 13(1), 22-40 (2016)

2. Bochev, P., Dohrmann, C., Gunzburger, M.: Stabilization of low-order mixed finite elements for the Stokes equations. SIAM J. Numer. Anal. 44, 82-101 (2006)

3. Braack, M., Burman, E., John, V., Lube, G.: Stabilized finite element methods for the generalized Oseen problem. Comput. Methods Appl. Mech. Eng. 196, 853-866 (2007)

4. Brezzi, F., Douglas, J.: Stabilized mixed methods for the Stokes problem. Numer. Math. 53, 225-235 (1988)

5. Brezzi, F., Pitkäanta, J.: On the stabilization of finite element approximation of the Stokes problem. In: Hackbush, W. (ed.) Efficient Solution of Elliptic Systems, pp. 11-19. Vieweg, Braunschweig (1984)

6. Burman, E., Fernandez, M.A., Hansbo, T.: Continuous interior penalty finite element method for Oseen's equations. SIAM J. Numer. Anal. 44, 1248-1274 (2006)

7. Chatzipantelidis, P., Makridakis, C., Plexousakis, M.: A-posteriori error estimates for a finite volume method for the Stokes problem in two dimensions. Appl. Numer. Math. 46, 45-58 (2003)

8. Chen, C.J., Zhao, X.: A posteriori error estimate for finite volume element method of the parabolic equations. Numer. Methods Partial Differ. Equ. 33(1), 259-275 (2017)

9. Chou, S., Li, Q.: Error estimates in $L^{2}, H^{1}$ and $L^{\infty}$ in covolume methods for elliptic and parabolic problem: a unified approach. Math. Comput. 229, 103-120 (2000)

10. Ciarlet, P.: The Finite Element Method for Elliptic Problems. North-Holland, Amsterdam (1978)

11. Codina, R.: Analysis of a stabilized finite element approximation of the Oseen equations using orthogonal subscales. Appl. Numer. Math. 58, 264-283 (2008)

12. Douglas, J., Wang, J.: An absolutely stabilized finite element method for the Stokes problem. Math. Comput. 52, 495-508 (1989)

13. Erath, C., Praetorius, D.: Adaptive vertex-centred finite volume methods with convergence rates. SIAM J. Numer. Anal. 54(4), 2228-2255 (2016)

14. Eymard, R., Gallouet, T., Herbin, R.: Finite volume methods. In: Ciarlet, P.G., Lions, J.L. (eds.) Handbook of Numerical Analysis, vol. 7, pp. 713-1020 (1997)

15. Eymard, R., Gutnic, M., Hilhorst, D.: The finite volume method for an elliptic-parabolic equation. Acta Math. Univ. Comen. LXVII(1), 181-195 (1998)

16. Girault, V., Raviart, P.A.: Finite Element Method for Navier-Stokes Equations: Theory and Algorithms. Springer, Berlin (1987)

17. He, Y.N., Li, J:: A stabilized finite element method based on local polynomial pressure projection for the stationary Navier-Stokes equations. Appl. Numer. Math. 58, 1503-1514 (2008)

18. Jing, F.F., Li, J., Chen, Z.X.: Numerical analysis of a characteristic stabilized finite element method for the time-dependent Navier-Stokes equations with nonlinear slip boundary conditions. J. Comput. Appl. Math. 320 43-60 (2017)

19. Kay, D., Silvester, D.: A posteriori error estimation for stabilized mixed approximations of the Stokes equations. SIAM J. Sci. Comput. 21, 1321-1336 (1999) 
20. Le Ha, A., Omnes, P.: An a posteriori error estimation for the discrete duality finite volume discretization of the Stokes equations. ESAIM: Math. Model. Numer. Anal. 49(3), 663-693 (2015)

21. Li, J., Chen, Z.X.: A new stabilized finite volume method for the stationary Stokes equations. Adv. Comput. Math. 30, $141-152(2009)$

22. Li, J., Chen, Z.X.: On the semi-discrete stabilized finite volume method for the transient Navier-Stokes equations. Adv. Comput. Math. 38, 281-320 (2013)

23. Li, J., Chen, Z.X., Zhang, T.: Adaptive stabilized finite volume methods for the incompressible flow. Numer. Methods Partial Differ. Equ. 31, 1424-1443 (2015)

24. Li, J., He, Y.N., Chen, Z.X.: A new stabilized finite element method for the transient Navier-Stokes equations. Comput. Methods Appl. Mech. Eng. 197, 22-35 (2007)

25. Li, R., Li, J., Chen, Z.X.: A stabilized finite element method based on two local Gauss integrations for a coupled Stokes-Darcy problem. J. Comput. Appl. Math. 292, 92-104 (2016)

26. Li, R.H., Chen, Z.Y., Wu, W.: Generalized Difference Methods for Differential Equations. Dekker, New York (2000)

27. Li, R.H., Zhu, P.Q.: Generalized difference methods for second order elliptic partial differential equations (I)-triangle grids. Numer. Math. J. Chinese Univ. 2, 140-152 (1982)

28. Li, Y., Li, J., Mei, L.Q.: Mixed stabilized finite element methods based on backward difference/Adams-Bashforth scheme for the time-dependent variable density incompressible flows. Comput. Math. Appl. 70(10), 2575-2588 (2015)

29. Lin, T., Ye, X:: A posteriori error estimates for finite volume method based on bilinear trial functions for the elliptic equation. J. Comput. Appl. Math. 254, 185-191 (2013)

30. Liu, J.G., Mu, L., Ye, X.: An adaptive discontinuous finite volume method for elliptic problems. J. Comput. Appl. Math 235(18), 5422-5431 (2011)

31. $\mathrm{Mu}, \mathrm{L} .$, Jari, R.: A posteriori error analysis for discontinuous finite volume methods of elliptic interface problems. J. Comput. Appl. Math. 255, 529-543 (2011)

32. Scott, L.R., Zhang, S.: Finite element interpolation of nonsmooth functions satisfying boundary conditions. Math. Comput. 54, 483-493 (1990)

33. Verfürth, R.: A Review of a Posteriori Error Estimation and Adaptive Mesh-Refinement Techniques. Wiley, New York (1996)

34. Wu, H.J., Li, R.H.: Error estimates for finite volume element methods for general second-order elliptic problems. Numer. Methods Partial Differ. Equ. 19, 693-708 (2003)

35. Ye, X:: On the relationship between finite volume and finite element methods applied to the Stokes equations. Numer. Methods Partial Differ. Equ. 5, 440-453 (2001)

36. Zhang, T., Mu, L., Yuan, J.Y: A posteriori error estimates of stabilized finite volume method for the Stokes equations. Math. Methods Appl. Sci. 39(1), 32-43 (2016)

37. Zhou, Y.H., Zou, Q.S.: A novel adaptive finite volume method for elliptic equations. Int. J. Numer. Anal. Model. 14(6), 879-892 (2017)

\section{Submit your manuscript to a SpringerOpen ${ }^{\circ}$ journal and benefit from:}

- Convenient online submission

- Rigorous peer review

- Open access: articles freely available online

- High visibility within the field

- Retaining the copyright to your article

Submit your next manuscript at $\gg$ springeropen.com 